THE ANALOGUE OF THE GAUSS PROBLEM IN MOTIVIC COHOMOLOGY 


\title{
THE ANALOGUE OF \\ THE GAUSS CLASS NUMBER PROBLEM IN MOTIVIC COHOMOLOGY
}

\author{
By \\ CAROLINE JUNKINS, B.SC.
}

A Thesis

Submitted to the School of Graduate Studies in Partial Fulfilment of the Requirements for the Degree

Master of Science

McMaster University

(C)Copyright by Caroline Junkins, June 2010 
MASTER OF SCIENCE (2010)

McMaster University

(Mathematics)

Hamilton, Ontario

TITLE: The Analogue of the Gauss Class Number Problem in Motivic Cohomology AUTHOR: Caroline Junkins, B.Sc. (McMaster University)

SUPERVISOR: Professor Manfred Kolster

NUMBER OF PAGES: vii, 56 


\begin{abstract}
Let $F$ be a totally real number field of degree $d$ and let $n \geq 2$ be an even integer. We denote by $W K_{2 n \rightarrow 2}^{\mathcal{M}}(F)$ the $n$-th motivic wild kernel of $F$, which acts as an analogue to the class group of $F$. Assuming the 2-adic Iwasawa Main Conjecture, we prove that the there are only finitely many totally real number fields $F$ having $\left|W K_{2 n-2}^{\mathcal{M}}(F)\right|=1$ for some even integer $n \geq 2$. In particular we show that there are no totally real number fields having trivial $n$-th motivic wild kernel for $n \geq 6$, and that there is precisely one totally real number field having trivial 4th motivic wild kernel, namely $\mathbb{Q}(\sqrt{5})$. We prove that all totally real number fields having trivial 2 nd motivic wild kernel must be of degree $d \leq 117$ (respectively $d \leq 46$ under the assumption of the Generalized Riemann Hypothesis). Using Sage mathematical software, we enumerate all totally real fields of degree $d<10$ having trivial 2 nd motivic wild kernel, finding 21 such fields. Under restrictions on the local properties of $F$, we enumerate all relevant fields having trivial 2nd motivic wild kernel.
\end{abstract}




\section{Acknowledgements}

I would first and foremost like to thank my advisor Manfred for his limitless supply of patience, guidance and humour. As both mentor and friend he has had a profound impact on my time at McMaster and my future in mathematics.

Thanks to Julia and Lina for always making me feel at home in Hamilton; I will definitely miss our movie/TV nights and your random visits to my office. Thanks as well to my great friends in Ottawa for their frequent trips to Hamilton and constant encouragement from home.

Finally I must thank my family and ski family for all of their help, love and support. Thank you so much for providing me with meals, visits and much-needed snowboarding time. Special thanks to my parents Bonny and Don, my sister Mellen, and my grandmother Dot. 


\section{Contents}

Descriptive Note . . . . . . . . . . . . . . . ii

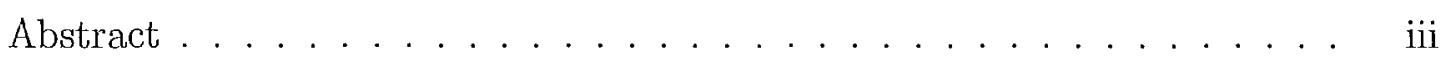

Acknowledgements . . . . . . . . . . . . . . iv

Introduction 1

1 Zeta Functions $\quad 6$

1.1 Riemann Zeta Function . . . . . . . . . . . . . . . . . 6

1.2 Dedekind Zeta Function $\ldots \ldots \ldots \ldots$

1.2.1 Estimating the values of the Dedekind Zeta function $\ldots \ldots 9$

2 Cohomology 11

2.1 Group Cohomology . . . . . . . . . . . . . . . . . . . . . . . . 12

2.1.1 Definition of Cohomology Groups . . . . . . . . . . . . 12

2.1 .2 The Standard Complex . . . . . . . . . . . . . . . . . . 14 
2.2 Galois Cohomology . . . . . . . . . . . . . . . 15

2.3 Étale Cohomology . . . . . . . . . . . . . 16

2.4 Motivic Cohomology . . . . . . . . . . . . . . . . 17

2.5 Motivic Class Number Formulae . . . . . . . . . . . . . . . . . . . 17

2.6 Totally Positive Cohomology . . . . . . . . . . . . . . 18

3 The Wild Kernel 22

3.1 Definition of Wild Kernel . . . . . . . . . . . . . . . 22

3.2 Poitou-Tate Duality Sequence . . . . . . . . . . . . . . . . 23

4 Number Fields of Bounded Discriminant $\quad 27$

4.1 Odlyzko Bounds . . . . . . . . . . . . . . . . 27

4.2 Enumeration of Totally Real Number Fields . . . . . . . . . . . . 29

4.2.1 Enumeration Process . . . . . . . . . . . . . 29

5 Main Theorem 32

5.1 Estimating $w_{n}(F) \ldots \ldots \ldots$. . . . . . . . . . . . . . . . . .

5.2 Estimating Local Factors . . . . . . . . . . . . . . . . . 33

5.3 Bounding the Root Discriminant . . . . . . . . . . . 35

5.4 Finding the Maximum Degree . . . . . . . . . . . . . . . 37 
5.4 .1 The Easy Case . . . . . . . . . . . . . . . . . . 38

5.4 .2 The Hard Case . . . . . . . . . . . . . . . . . . . . . . 39

5.5 Enumerating the Fields . . . . . . . . . . . . . . . . . . . . 42

6 Some Special Cases 44

6.1 Assuming GRH $\ldots \ldots \ldots \ldots \ldots \ldots \ldots$

6.2 Restricting Local Factors . . . . . . . . . . . . . . . . . . 45

$\begin{array}{lll}7 & \text { Computations } & 49\end{array}$

$7.1 \quad$ Enumerating $\mathcal{N F}_{a}(n) \quad \ldots \ldots \ldots \ldots \ldots \ldots \ldots$

7.2 Results . . . . . . . . . . . . . . . . . . . 51

Bibliography $\quad 54$ 


\section{Introduction}

In Article 303 of Disquisitiones Arithmeticae, Gauss first posed the problem of determining all imaginary quadratic fields $F$ of small class number $h_{F}$, and in particular, all such fields having $h_{F}=1$.

Let $F$ be a number field of degree $d=r_{1}+2 r_{2}$, where $r_{1}, r_{2}$ denote the number of real places and pairs of complex conjugate places respectively. We denote by $\zeta_{F}(s)$ the Dedekind zeta function of $F$. The Dedekind zeta function admits a functional equation relating the vales $\zeta_{F}(s)$ and $\zeta_{F}(1-s)$. This functional equation gives the result that for an integer $n \geq 1$, the order of vanishing of $\zeta_{F}(s)$ at $s=1-n$, denoted $d_{n}$, is equal to

$$
d_{n}= \begin{cases}r_{1}+r_{2}-1 & \text { if } n=1 \\ r_{1}+r_{2} & \text { if } n \geq 3 \text { is odd } \\ r_{2} & \text { if } n \geq 2 \text { is even }\end{cases}
$$

Thus it can be seen that the value of $\zeta_{F}(0)$ is non-zero precisely when $F=\mathbb{Q}$ or $F$ is an imaginary quadratic field.

For such a number field $F$, Dirichlet's Analytic Class Number Formula provides a relation between the value $\zeta_{F}(0)$ of the zeta-function and the class number $h_{F}$, given by

$$
\zeta_{F}(0)=-\frac{h_{F}}{w_{F}},
$$

where $w_{F}$ denotes the number of roots of unity in $F$. A complete list of imaginary quadratic number fields $F$ having $h_{F}=1$ was determined by Stark in 1967 ([24]). In 2004, Watkins computed a complete list of all imaginary quadratic number fields with class number $\leq 100([30])$.

An analogue to the Gauss class number problem arises if we consider the values 
$\zeta_{F}(1-n)$, for even integers $n \geq 2$. Again by the functional equation, it is clear that the value of $\zeta_{F}(1-n)$ is non-zero precisely whon $n \geq 2$ is even and $F$ is totally real. In this situation, the value of $\zeta_{F}(1-n)$ was proven to be rational by Klingen and Siegel ([7], [23]).

As a consequence of the Main Conjecture of Iwasawa Theory, this value is given by

Theorem. (Wiles) Assume $F$ is a totally real number field and that $n \geq 2$ is an even integer. Then

$$
\zeta_{F}(1-n)=(-1)^{\frac{n}{2}} \frac{h_{n}(F)}{w_{n}(F)},
$$

where $h_{n}(F)$ denotes the order of the motivic cohomology group $H_{\mathcal{M}}^{2}\left(o_{F}, \mathbb{Z}(n)\right)$ of the ring of integers $o_{F}$ of $F$, and $w_{n}(F)$ denotes the order of the Galois cohomology group $H^{0}(F, \mathbb{Q} / \mathbb{Z}(n))$. We note that for $n=1$, the group $H^{0}(F, \mathbb{Q} / \mathbb{Z}(1))$ consists of the roots of unity in $F$, and hence $w_{1}(F)=w_{F}$ in our previous notation.

The Iwasawa Main Conjecture has been proven by Wiles (cf. [31]) for odd primes $p$. The 2-primary part of the conjecture has been proven by Wiles for abelian number fields, but remains open in general. The general 2-adic Iwasawa main conjecture will be assumed throughout the paper.

The analogue of the Gauss class number problem is to determine all totally real number fields $F$ having $h_{n}(F)=1$ for even integers $n \geq 2$. It can be shown that if $F$ is of degree $d=[F: \mathbb{Q}]$, then $h_{n}(F) \geq 2^{d}$, and hence can never be trivial. A better candidate for the analogue of the class number is therefore given by the order $h_{n}^{\mathcal{M}}(F)$ of $W K_{2 n-2}^{\mathcal{M}}(F)$, the $n$-th motivic wild kernel of $F$, which is a canonical subgroup of $H_{\mathcal{M}}^{2}\left(o_{F}, \mathbb{Z}(n)\right)$ determined by local conditions. This subgroup is analogous to the Tate-Shafarevich group of an abelian variety $A$ defined over a number field $F$.

The functional equation of the Dedekind zeta function relates the values $\zeta_{F}(s)$ and $\zeta_{F}(1-s)$ to the discriminant $D$ of $F$. For a totally real number field $F$ and an even integer $n \geq 2$, we then obtain the inequality

$$
D^{n-\frac{1}{2}} \leq\left|\zeta_{F}(1-n)\right| \cdot\left(\frac{(2 \pi)^{n}}{2(n-1) !}\right)^{d},
$$

where $d=[F: \mathbb{Q}]$. 
The above theorem of Wiles is used to restate this inequality in terms of cohomology groups:

$$
D^{n-\frac{1}{2}} \leq \frac{h_{n}(F)}{w_{n}(F)}\left(\frac{(2 \pi)^{n}}{2(n-1) !}\right)^{d} .
$$

The index of $W K_{2 n-2}^{\mathcal{M}}(F)$ in $H_{\mathcal{M}}^{2}\left(o_{F}, \mathbb{Z}(n)\right)$ is determined by local conditions and can be estimated as follows:

$$
\frac{h_{n}(F)}{h_{n}^{\mathcal{M}}(F)} \leq 2^{d(d-1)}(n-1)^{d / 2} D .
$$

Combining these two estimates gives the following bound on the root discriminant $\delta_{F}$ of $F$, defined as $\delta_{F}=D^{1 / d}$,

$$
\delta_{F} \leq\left(\frac{2(2 \pi)^{n}}{(n-1)^{1 / 2}(n-2) !}\left(\frac{h_{n}^{\mathcal{M}}(F)}{2 w_{n}(F)}\right)^{1 / d}\right)^{\frac{2}{2 n-3}} .
$$

Therefore, for any positive integer $a$, all totally real number fields $F$ having $h_{n}^{\mathcal{M}}(F)=a$ must be of bounded root discriminant.

The subject of number fields of bounded root discriminant dates back to Minkowski, who determined the first discriminant bounds geometrically (cf. [15]). Through analytic methods, Odlyzko has developed a set of lower bounds $B_{O}(d)$ defined such that for all totally real number fields $F$ of degree $d, \delta_{F}>B_{O}(d)$ ([17]). Using these Odlyzko bounds, Voight has developed a process to enumerate totally real number fields of bounded root discriminant, and has provided lists of all totally real number fields $F$ having $\delta_{F}<14([27])$.

By comparing our estimates with the Odlyzko bounds, it can be seen that our above bound is quite effective for even integers $n \geq 4$, and eliminates all but a very small number of possible fields. However, for the classical case $n=2$ the bound does not yield a reasonable set of possible fields, and so we consider these two cases separately:

We establish that the set of totally real number fields $F$ having $h_{n}^{\mathcal{M}}(F)=a$ is finite for all even integers $n \geq 4$ and all positive integers $a \leq 32$. As well, we use the 
enumeration process described by Voight to show that in the case $n \geq 4$ there is preciscly one totally real number field $F$ having $h_{4}^{\mathcal{M}}(F)=1$, namely the field $F=\mathbb{Q}(\sqrt{5})$.

For the case $n=2$, we modify the upper bound on $\delta_{F}$ through improved estimates using the local properties of $F$. With this modified bound, we are able to prove that the set of totally real number fields having $h_{2}^{\mathcal{M}}(F)=1$ is finite as well, and contains only fields of degree $d \leq 117$.

Combining these results yields our main theorem:

Theorem. There are finitely many totally real number fields $F$ such that $h_{n}^{\mathcal{M}}(F)=1$ for some even integer $n \geq 2$.

Through computations performed in Sage and Pari/GP ([25], [19]), we enumerate all totally real number fields $F$ of degree $d<10$ having $h_{2}^{\mathcal{M}}(F)=1$. Within this list, we find no number fields of degree $d>5$, and we predict that only isolated cases of higher degree may exist. We present two special cases to support this prediction.:

Under the assumption of the Generalized Riemann Hypothesis, we show that the set of all totally real number fields $F$ having $h_{2}^{\mathcal{M}}(F)=a$ is finite for all positive integers $a \leq 756$, and that $h_{2}^{\mathcal{M}}(F)>1$ if $[F: \mathbb{Q}] \geq 47$.

For our second special case, we let $F$ be a totally real number field which contains at most unramified local cyclotomic extensions. That is, for all odd primes $p$ and all $v \mid p$ in $F, \mathbb{Q}_{p}\left(\zeta_{p}\right) \nsubseteq F_{v}$, and for all $v \mid 2, \mathbb{Q}_{2}(i) \nsubseteq F_{v}$. Under this assumption, the index of $W K_{2}^{\mathcal{M}}(F)$ in $H_{\mathcal{M}}^{2}\left(o_{F}, \mathbb{Z}(2)\right)$ is given by

$$
\frac{h_{2}(F)}{h_{2}^{\mathcal{M}}\left(F^{\prime}\right)}=2^{\#(v \mid 2)+d-1},
$$

where \#(v|2) denotes the number of primes above 2 in $F$. Thus, we obtain an improved bound on $\delta_{F}$ via the functional equation of $\zeta_{F}(s)$,

$$
\delta_{F} \leq\left(\frac{2(2 \pi)^{2}}{\left(48 \cdot \zeta_{F}(2)\right)^{1 / d}}\right)^{2 / 3},
$$

This bound is sufficient to show that $h_{2}^{\mathcal{M}}(F)>1$ if $[F: \mathbb{Q}]>9$, and we obtain via our previous computations that there are precisely 14 such number fields having $h_{2}^{\mathcal{M}}(F)=1$. 
In Chapter 1 we review the theory of zeta functions. Chapter 2 provides the necessary cohomological background, while Chapter 3 introduces the motivic wild kernel. In Chapter 4 we summarize research done by Odlyzko, Voight and others concerning minimal root discriminants of number fields and the enumeration of number fields of bounded discriminant. The main results of our thesis are presented in Chapter 5, and some special cases are discussed in Chapter 6. Finally, Chapter 7 provides the algorithms used to compute $h_{n}^{\mathcal{M}}(F)$ and the results of such computations. 


\section{Chapter 1}

\section{Zeta Functions}

We begin this Chapter by introducing the Riemann zeta function and some of its important properties in Section 1.1. Once established, Section 1.2 describes a generalization of this function, called the Dedekind zeta function.

The theory of zeta functions presented in this paper is taken primarily from [15].

\subsection{Riemann Zeta Function}

The Riemann zeta function is defined by the infinite series

$$
\zeta(s)=\sum_{n=1}^{\infty} \frac{1}{n^{s}}
$$

where $s$ is a complex variable. This series is absolutely and uniformly convergent on the domain $\operatorname{Re}(s) \geq 1+\delta$, for every $\delta>0$, and hence represents an analytic function in the half-plane $R e(s)>1$. On this half plane, the Riemann zeta function can also be represented by Euler's identity

$$
\zeta(s)=\prod_{p} \frac{1}{1-p^{-s}},
$$

where $p$ runs through the prime numbers. 
The Riemann zeta function admits a meromorphic continuation throughout the complex plane with a single simple pole at $s=1$ and residue

$$
\lim _{s \rightarrow 1}(s-1) \zeta(s)=1 .
$$

Furthermore, it satisfies a functional equation, which relates the values at $s$ and $1-s$ :

$$
\pi^{-\frac{s}{2}} \Gamma\left(\frac{s}{2}\right) \zeta(s)=\pi^{\frac{s-1}{2}} \Gamma\left(\frac{1-s}{2}\right) \zeta(1-s),
$$

where $\Gamma(s)$ denotes the $\Gamma$-function, a meromorphic function having simple poles at $s=-m, m=0,1,2, \ldots$, with residues $(-1)^{m} / m !$.

\subsection{Dedekind Zeta Function}

The Riemann zeta function is associated to the field of rational numbers, but can be generalized to an arbitrary number field $F$ of degree $d=[F: \mathbb{Q}]$. This generalization is called the Dedekind zeta function and is defined by

$$
\zeta_{F}(s)=\sum_{0 \neq I \subset o_{F}} \frac{1}{N(I)^{s}},
$$

where $o_{F}$ denotes the ring of integers in $F$, and for any non-zero ideal $I \subset o_{F}$, $N(I)=\left|o_{F} / I\right|$ denotes the number of elements in the finite quotient ring $o_{F} / I$. This series again represents an analytic function on the half-plane $\operatorname{Re}(s)>1$, and has the identity

$$
\zeta_{F}(s)=\prod_{\mathfrak{p}} \frac{1}{1-N(\mathfrak{p})^{-s}},
$$

where $\mathfrak{p}$ runs through all non-zero prime ideals of $F$.

The Dedekind zeta function also admits a meromorphic continuation throughout the complex plane with a single simple pole at $s=1$.

The residue of the Dedekind zeta function at $s=1$ is given by

$$
\lim _{s \rightarrow 1}(s-1) \zeta_{F}(s)=\frac{2^{r_{1}} \cdot(2 \pi)^{r_{2}} \cdot h_{F} \cdot R_{F}}{w_{F} \cdot \sqrt{|D|}}
$$


where $h_{F}$ is the class number of $F, R_{F}$ is the regulator of $F$ and $w_{F}$ denotes the number of roots of unity in $F$.

The Dedekind zeta function satisfies a functional equation relating the values at $s$ and $1-s$. Let

$$
A=2^{-r_{2}} \pi^{-\frac{d}{2}} \sqrt{|D|}
$$

where $D$ denotes the discriminant of $F$, and $d=r_{1}+2 r_{2}$ (where $r_{1}, r_{2}$ denote the number of real places and pairs of complex conjugate places respectively). Then the functional equation reads

$$
A^{s} \Gamma\left(\frac{s}{2}\right)^{r_{1}} \Gamma(s)^{r_{2}} \zeta_{F}(s)=A^{1-s} \Gamma\left(\frac{1-s}{2}\right)^{r_{1}} \Gamma(1-s)^{r_{2}} \zeta_{F}(1-s) .
$$

The functional equation implies that the order of vanishing of $\zeta_{F}(s)$ at $s=1-n$, denoted $d_{n}$, is equal to

$$
d_{n}= \begin{cases}r_{1}+r_{2}-1 & \text { if } n=1 \\ r_{1}+r_{2} & \text { if } n \geq 3 \text { is odd } \\ r_{2} & \text { if } n \geq 2 \text { is even }\end{cases}
$$

If we denote by $\zeta_{F}^{*}(s)$ the first non-vanishing coefficient in a Taylor expansion of the Dedekind zeta function around $s$ and call this the special value of the zeta function at $s$, then the residue (1.2) yields an important result of Dirichlet, called the Analytic Class Number Formula:

Theorem 1.1. (Dirichlet) For a number field $F$,

$$
\zeta_{F}^{*}(0)=-\frac{h_{F}}{w_{F}} \cdot R_{F} .
$$

In the special case that $F$ is an imaginary quadratic field, we see from equation (1.4) that the Dedekind zeta function of $F$ does not vanish at $s=0$, and hence $\zeta_{F}^{*}(0)=\zeta_{F}(0)$ in this case. Thus,

$$
\zeta_{F}(0)=-\frac{h_{F}}{w_{F}} .
$$


The Analytic Class Number Formula is a deep result connecting a special value of the Dedekind zeta function of a number field $F$ to the class number of $F$. As can be expected, this result can be extended to other special values of the zeta function, which can in turn be related to other important arithmetic properties of $o_{F}$. We will return to one of these results after developing the necessary background in the next chapter.

\subsubsection{Estimating the values of the Dedekind Zeta function}

For a totally real number field $F$ and an even integer $n \geq 2$, equation (1.4) implies that $\zeta_{F}(s)$ does not vanish at $s=1-n$, and hence $\zeta_{F}^{*}(1-n)=\zeta_{F}(1-n)$. This value can be estimated via the functional equation, which in this specific case is given by:

Lemma 1.2. Let $F$ be a totally real number field of degree $d$ and discriminant $D$, and let $n \geq 2$ be an even integer. Then

$$
(-1)^{\frac{d n}{2}} \zeta_{F}(1-n)=D^{n-\frac{1}{2}} \cdot\left(\frac{2(n-1) !}{(2 \pi)^{n}}\right)^{d} \cdot \zeta_{F}(n) .
$$

Proof. Since $F$ is totally real, (1.3) reads

$$
D^{\frac{s}{2}}\left(\pi^{\frac{-s}{2}} \Gamma\left(\frac{s}{2}\right)\right)^{d} \cdot \zeta_{F}(s)=D^{\frac{1-s}{2}}\left(\pi^{\frac{s-1}{2}} \cdot \Gamma\left(\frac{1-s}{2}\right)\right)^{d} \cdot \zeta_{F}(1-s) .
$$

Let $s=n=2 m$. Then $\Gamma\left(\frac{n}{2}\right)=\Gamma(m)=(m-1)$ ! and as a consequence of Legendre's duplication formula,

$$
\Gamma\left(\frac{1-n}{2}\right)=\Gamma\left(\frac{1}{2}-m\right)=(-1)^{m} \frac{2^{2 m-1} m !}{(2 m) !} \cdot \sqrt{\pi}=(-1)^{m} \frac{2^{2 m}(m-1) !}{2(2 m-1) !} \cdot \sqrt{\pi} .
$$

The result follows.

For real $s>1$, we have that $\zeta_{F}(s) \geq 1$, and

$$
\zeta_{F}(s) \rightarrow 1 \quad \text { as } \quad s \rightarrow \infty \text {. }
$$


Since $n$ is defined to be a positive integer, taking absolute values in the above equation and replacing $\zeta_{F}(n)$ by 1 will yield a valid lower bound on the values of the Dedekind zeta function of $F$.

\section{Corollary 1.3.}

$$
\left|\zeta_{F}(1-n)\right| \geq D^{n-\frac{1}{2}} \cdot\left(\frac{2(n-1) !}{(2 \pi)^{n}}\right)^{d} .
$$




\section{Chapter 2}

\section{Cohomology}

The values of the Dedekind zeta function $\zeta_{F}(s)$ of a totally real number field $F$ at odd negative integers are non-zero rational numbers ([23]). An interpretation of the value at $s=-1$ was suggested by Birch and Tate in 1970 in terms of algebraic Ktheory, and was generalized to all number fields and negative integers by Lichtenbaum.

The "correct" version of these Lichtenbaum conjectures should involve motivic cohomology rather than K-theory, the difference being of 2-power order. We present in Sections 2.1 to 2.4 the necessary cohomological background. In Section 2.5 we discuss the Lichtenbaum Conjecture in the special case of totally real number fields $F$ and values of $\zeta_{F}(s)$ at negative integers. Finally in section 2.6 we present a reformulation of the conjecture using totally positive cohomology groups.

The cohomology theory presented here is taken primarily from [22], [15] and [1]. 


\subsection{Group Cohomology}

\subsubsection{Definition of Cohomology Groups}

Let $\mathcal{C}$ be a sequence of abelian groups or modules connected by group homomorphisms $d_{i}: C^{i-1} \rightarrow C^{i}$

$$
0 \longrightarrow C^{0} \stackrel{d_{1}}{\longrightarrow} C^{1} \longrightarrow \cdots \longrightarrow C^{n-1} \stackrel{d_{n}}{\longrightarrow} C^{n} \stackrel{d_{n+1}}{\longrightarrow} \cdots
$$

The sequence $\mathcal{C}$ is called a cochain complex if the composition of any two maps is zero, i.e. $d_{n+1} \circ d_{n}=0$ for all $n$. It follows that $i m\left(d_{n}\right) \subseteq \operatorname{ker}\left(d_{n+1}\right)$ for all $n$, hence we can define the $n^{\text {th }}$ cohomology group of $\mathcal{C}$ to be the quotient group $H^{n}(\mathcal{C})=\operatorname{ker}\left(d_{n+1}\right) / i m\left(d_{n}\right)$. It is clear that the sequence $\mathcal{C}$ is exact if and only if $H^{n}(\mathcal{C})=0$ for all $n$. Thus, the $n^{t h}$ cohomology group can be interpreted as a measurement of the failure of exactness of $\mathcal{C}$ at the $n$th stage.

Consider a group $G$ with its integral group $\operatorname{ring} \Lambda=\mathbb{Z}[G]$. A G-module $A$ is the same as a $\Lambda$-module. If $A, B$ are $G$-modules, the group of all group homomorphims $A \rightarrow B$ is denoted $\operatorname{Hom}(A, B)$, while the group of all $G$-module homomorphisms $A \rightarrow B$ is denoted $\operatorname{Hom}_{G}(A, B)$. The subset of elements of $A$ which are invariant under the action of $G$ is denoted $A^{G}$. As well, we have the equality

$$
\operatorname{Hom}_{G}(A, B)=(\operatorname{Hom}(A, B))^{G},
$$

and in particular,

$$
\operatorname{Hom}_{G}(\mathbb{Z}, A)=(\operatorname{Hom}(\mathbb{Z}, A))^{G} \cong A^{G},
$$

where $\mathbb{Z}$ is regarded as a $G$-module on which $G$ acts trivially. Since $H o m$ is a left-exact functor, we can view $A \mapsto A^{G}$ as a left-exact functor taking $G$-modules to abelian groups. Hence, if

$$
0 \longrightarrow A \longrightarrow B \longrightarrow C \longrightarrow 0
$$

is an exact sequence of $G$-modules, then

$$
0 \longrightarrow A^{G} \longrightarrow B^{G} \longrightarrow C^{G}
$$

is an exact sequence of abelian groups which does not, in general, terminate with a surjective map.

By constructing a cohomological extension of the functor $A \mapsto A^{G}$ we form the 
right derived functors denoted by $I^{n}(G, A)$, with $H^{0}(G, A)=A^{G}$. These functors are called the cohomology groups of $G$ with coefficients in $A$. To construct these functors we first need the notion of a projective resolution.

Considering $\mathbb{Z}$ as a $G$-module, a projective resolution is an exact sequence

$$
\cdots \longrightarrow P_{n} \stackrel{d_{n}}{\longrightarrow} P_{n-1} \longrightarrow \cdots \stackrel{d_{1}}{\longrightarrow} P_{0} \stackrel{\epsilon}{\longrightarrow} \mathbb{Z} \longrightarrow 0
$$

such that each $P_{i}$ is a projective $G$-module. From this, we obtain a cochain complex of $G$-module homomorphisms from the projective resolution into $A$,

$$
0 \longrightarrow \operatorname{Hom}_{G}(\mathbb{Z}, A) \stackrel{\epsilon}{\longrightarrow} \operatorname{Hom}_{G}\left(P_{0}, A\right) \stackrel{d_{1}}{\longrightarrow} \cdots \stackrel{d_{n-1}}{\longrightarrow} \operatorname{Hom}_{G}\left(P_{n-1}, A\right) \stackrel{d_{n}}{\longrightarrow} \cdots
$$

keeping in mind that this reverses the direction of the homomorphisms. The first four terms of the sequence

$$
0 \longrightarrow \operatorname{Hom}_{G}(\mathbb{Z}, A) \stackrel{\epsilon}{\longrightarrow} \operatorname{Hom}_{G}\left(P_{0}, A\right) \stackrel{d_{1}}{\longrightarrow} \operatorname{Hom}_{G}\left(P_{1}, A\right)
$$

are exact, and hence

$$
\operatorname{ker}\left(d_{1}\right)=i m(\epsilon) \cong \operatorname{Hom}_{G}(\mathbb{Z}, A) \cong A^{G} .
$$

Replacing $\operatorname{Hom}_{G}(\mathbb{Z}, A)$ by 0 does not affect the cochain property of the sequence, which can then be written as

$$
0 \longrightarrow \operatorname{Hom}_{G}\left(P_{0}, A\right) \stackrel{d_{1}}{\longrightarrow} \operatorname{Hom}_{G}\left(P_{1}, A\right) \stackrel{d_{2}}{\longrightarrow} \cdots \stackrel{d_{n-1}}{\longrightarrow} \operatorname{Hom}_{G}\left(P_{n-1}, A\right) \stackrel{d_{n}}{\longrightarrow} \cdots
$$

The cohomology groups defined by $H^{n}(G, A)=\operatorname{ker}\left(d_{n+1}\right) / i m\left(d_{n}\right)$ of this sequence do not depend on the choice of projective resolution, and satisfy the required properties to be a cohomological extension of the functor $A \mapsto A^{G}$. That is, $H^{0}(G, A)=A^{G}$, and for any exact sequence

$$
0 \rightarrow A \rightarrow B \longrightarrow C \longrightarrow 0
$$

we can define connecting (or boundary) homomorphisms

$$
\delta: H^{n}(G, C) \rightarrow H^{n+1}(G, A)
$$

resulting in a long exact sequence

$$
\cdots \longrightarrow H^{i}(G, A) \longrightarrow H^{i}(G, B) \longrightarrow H^{i}(G, C) \stackrel{\delta}{\longrightarrow} H^{i+1}(G, A) \longrightarrow \cdots
$$




\subsubsection{The Standard Complex}

Since the cohomology groups are independent of the choice of projective resolution, we are able to work with a particular choice of resolution $P$, called the standard resolution. Let $P_{i}=\mathbb{Z}\left[G^{i+1}\right]$, i.e. $P_{i}$ is the free $\mathbb{Z}$-module with basis $G \times \cdots \times G$ (with $i+1$ factors), where an element $s \in G$ acts on each basis element by

$$
s\left(g_{0}, g_{1}, \ldots, g_{i}\right)=\left(s g_{0}, s g_{1}, \ldots, s g_{i}\right) .
$$

The homomorphism $d_{i}: P_{i} \rightarrow P_{i-1}$ is given by

$$
d_{i}\left(g_{o}, \ldots, g_{i}\right)=\sum_{j=0}^{i}(-1)^{j}\left(g_{o}, \ldots, g_{j-1}, g_{j+1}, \ldots, g_{i}\right) .
$$

The elements of $\operatorname{Hom}_{G}\left(P_{i}, A\right)$ are functions $f: G^{i+1} \rightarrow A$ such that

$$
f\left(s g_{o}, s g_{1}, \ldots, s g_{i}\right)=s \cdot f\left(g_{0}, g_{1}, \ldots, g_{i}\right) .
$$

These elements form a cochain complex with boundary homomorphisms $d: H_{0} m_{G}\left(P_{i-1}, A\right) \rightarrow$ $H_{0 m}\left(P_{i}, A\right)$ given by

$$
\begin{aligned}
d(f)\left(g_{1}, \ldots, g_{i+1}\right)= & g_{1} \cdot f\left(g_{2}, \ldots, g_{i+1}\right) \\
& +\sum_{j=1}^{i}(-1)^{j} f\left(g_{1}, \ldots, g_{j-1}, g_{j} g_{j+1}, g_{j+2}, \ldots, g_{i+1}\right) \\
& +(-1)^{i+1} f\left(g_{1}, \ldots, g_{i}\right)
\end{aligned}
$$

We can verify that these definitions satisfy the required property that $H^{0}(G, A)=$ $A^{G}$. We note $H^{0}(G, A)=\operatorname{ker}(d)$, and $\operatorname{Hom}_{G}(G, A)$ is given by all constant maps $f=a, a \in A$. By $(2.1)$, we have

$$
(d f)(g)=g \cdot a-a,
$$

so $f \in \operatorname{ker}(d)$ if and only if $g \cdot a=a$, hence if and only if $a \in A^{G}$.

When discussing the cohomology groups of $\mathrm{G}$ without specifying a $G$-module $A$, we write $H^{n}(G, \bullet)$. 


\subsection{Galois Cohomology}

An application of group cohomology arises when we consider $G$ to be the Galois group of a field extension $K / F$. In particular, we can choose $K=\bar{F}$, the separable closure of $F$. The Galois group $G_{F}=\operatorname{Gal}(\bar{F} / F)$ is the inverse limit $\lim _{\longleftrightarrow} \operatorname{Gal}(L / F)$ of the Galois groups of the finite extensions $L$ of $F$ contained in $\bar{F}$, and is a compact topological group. A discrete $G_{F}$-module $A$ is a $G_{F}$-module with the discrete topology such that the action of $G_{F}$ on $A$ is continuous. The cohomology groups $H^{i}\left(G_{F}, A\right)$ computed using continuous cochains are called the Galois cohomology groups of $F$, and are denoted simply by $H^{i}(F, A)$.

Let $F$ be a number field, and fix a prime $p$. Let $\mu_{p^{m}}$ denote the group of $p^{m}$-th roots of unity, and let $\mu_{p^{m}}^{\otimes n}$ be the $n$-fold tensor product of this group. We define the projective limit

$$
\mathbb{Z}_{p}(n)=\varliminf_{\longleftrightarrow} \mu_{p^{m}}^{\otimes n}
$$

and the injective limit

$$
\mathbb{Q}_{p} / \mathbb{Z}_{p}(n)=\mu_{p^{\infty}}^{\otimes n}=\lim _{\longrightarrow} \mu_{p^{m}}^{\otimes n} .
$$

Defining diagonal $G$-action on $\mu_{p^{n}}^{\otimes n}$ by $g(\zeta \otimes \cdots \otimes \zeta)=g(\zeta) \otimes \cdots \otimes g(\zeta)$, we can examine the elements of $\mu_{p^{\infty}}^{\otimes n}$ invariant under the action of $G_{F}=G a l\left(F\left(\mu_{p^{\infty}}\right) / F\right)$. These elements are precisely those contained in the Galois cohomology group

$$
H^{0}\left(F, \mathbb{Q}_{p} / \mathbb{Z}_{p}(n)\right)=\underline{\lim _{\longrightarrow}} H^{0}\left(F, \mu_{p^{m}}^{\otimes n}\right) .
$$

To find the order of this group, we look more closely at the action of $G$ on the tensor product. If $g \in \operatorname{Gal}\left(F\left(\mu_{p^{\infty}}\right) / F\right)$, then $g(\zeta)=\zeta^{a}$ for some integer $a$. Hence,

$$
\begin{aligned}
g\left(\zeta^{\otimes n}\right) & =g(\zeta) \otimes \cdots \otimes g(\zeta) \\
& =\zeta^{a} \otimes \cdots \otimes \zeta^{a} \\
& =\left(\zeta^{\otimes n}\right)^{a^{n}} .
\end{aligned}
$$

In other words, the action of $g$ on $\mu_{p^{m}}^{\otimes n}$ is identical to the action of $g^{n}$ on $\mu_{p^{m}}$. So, $\mu_{p^{m}}^{\otimes n}$ is fixed under $\operatorname{Gal}\left(F\left(\mu_{p^{m}}\right) / F\right)$ precisely when this group is of exponent $n$ (in 
the sense that for all $g \in G, g^{n}=1$ ). Relating this to the previously defined Galois cohomology groups, we have

$$
\left|H^{0}\left(F, \mathbb{Q}_{p} / \mathbb{Z}_{p}(n)\right)\right|=\left\{\max p^{m}: \operatorname{Gal}\left(F\left(\mu_{p^{m}}\right) / F\right) \text { has exponent } n\right\} .
$$

F'inally we define

$$
H^{0}(F, \mathbb{Q} / \mathbb{Z}(n))=\prod_{p} H^{0}\left(F, \mathbb{Q}_{p} / \mathbb{Z}_{p}(n)\right)
$$

where $p$ runs through all primes. We denote the order of this finite group by $w_{n}(F)$.

Remark 1. For a totally real field $F$, the Galois group $\operatorname{Gal}\left(F\left(\mu_{p^{m}}\right) / F\right)$ must contain an element of order 2 corresponding to complex conjugation. Thus, for any odd integer $n \geq 1$, we have $w_{n}(F)=2$.

\section{$2.3 \quad$ Étale Cohomology}

Motivated by the desire to prove the Weil Conjectures, Grothendieck defined étale cohomology geometrically for schemes with values in certain sheaves. In our situation the scheme will be the affine scheme $\operatorname{spec}(R)$ for a certain Dedekind ring $R$, and the sheaves will consist of roots of unity. Milne ([13], ex. 3.16) shows that in this case, étale cohomology can be interpreted as a certain Galois cohomology, described as follows:

Let $F$ be a number field with ring of integers $o_{F}$ and let $p$ be a prime number. Let $o_{F}^{\prime}=o_{F}\left[\frac{1}{p}\right]$. The étale cohomology groups of $\operatorname{spec}\left(o_{F}^{\prime}\right)$ with values in the $n$-fold twisted sheaf of $p^{m}$-th roots of unity are isomorphic to the Galois cohomology groups $I^{i}\left(G_{F}^{(p)}, \mu_{p^{m}}^{\otimes n}\right)$, where $G_{F}^{(p)}=\operatorname{Gal}\left(\Omega_{F}^{(p)} / F\right)$. We will denote these groups simply by $H_{\mathrm{ett}}^{i}\left(o_{F}^{\prime}, \mu_{p^{m}}^{\otimes n}\right)$.

Following the previous notation, we define the $p$-adic cohomology groups

$$
H_{\text {êt }}^{i}\left(o_{F}^{\prime}, \mathbb{Z}_{p}(n)\right)=\lim _{\longleftarrow} H_{\text {ét }}^{i}\left(o_{F}^{\prime}, \mu_{p^{m}}^{\otimes n}\right)
$$

and

$$
H_{\mathrm{ét}}^{i}\left(o_{F}^{\prime}, \mathbb{Q}_{p} / \mathbb{Z}_{p}(n)\right)=\lim _{\longrightarrow} H_{\mathrm{ét}}^{i}\left(o_{F}^{\prime}, \mu_{p^{m}}^{\otimes n}\right)
$$

From [9], we have the following properties: 
- For $n \neq 0, H_{\text {ét }}^{0}\left(o_{F}^{\prime}, \mathbb{Z}_{p}(n)\right)=0$

- For $n \geq 2, r k_{\mathbb{Z}_{p}} H_{\text {ét }}^{1}\left(o_{F}^{\prime}, \mathbb{Z}_{p}(n)\right)= \begin{cases}r_{1}+r_{2} & \text { if } n \text { is odd } \\ r_{2} & \text { if } n \text { is even }\end{cases}$

- For $n \geq 2, H_{\text {ét }}^{2}\left(o_{F}^{\prime}, \mathbb{Z}_{p}(n)\right)$ is finite and trivial for almost all primes $p$

- For $i \geq 3: H_{\text {ét }}^{i}\left(o_{F}^{\prime}, \mathbb{Z}_{p}(n)\right)=0$ if $p$ is odd

- For $i \geq 3: H_{\mathrm{ét}}^{i}\left(o_{F}^{\prime}, \mathbb{Z}_{2}(n)\right) \cong \begin{cases}(\mathbb{Z} / 2 \mathbb{Z})^{r_{1}} & \text { if } i+n \text { is even } \\ 0 & \text { otherwise }\end{cases}$

\subsection{Motivic Cohomology}

While the $p$-adic cohomology groups are defined for a single prime $p$, we would like to view them as the $p$-parts of a 'global' cohomology. The two candidates for this 'global' cohomology are Algebraic K-Theory and Motivic Cohomology. The BlochKato conjecture, which has been proved by Rost and Voevodsky implies that there are isomorphisms

$$
K_{2 n-i}\left(o_{F}\right) \cong H_{\mathcal{M}}^{i}\left(o_{F}, \mathbb{Z}(n)\right)
$$

up to 2 -torsion for all $n \geq 2$ and $i=1,2$. In general, the 2 -torsion is different, and it is motivic cohomology which has the property that

$$
H_{\mathcal{M}}^{i}\left(o_{F}, \mathbb{Z}(n)\right) \otimes \mathbb{Z}_{p} \cong H_{\text {ét }}^{i}\left(o_{F}^{\prime}, \mathbb{Z}_{p}(n)\right)
$$

for all primes $p$.

We will be particularly interested in the $2^{\text {nd }}$ motivic cohomology groups $H_{\mathcal{M}}^{2}\left(o_{F}, \mathbb{Z}(n)\right)$ of $o_{F}$ for $n \geq 2$. These groups are finite, as a consequence of the properties of the corresponding étale cohomology groups listed in Section 2.3.

\subsection{Motivic Class Number Formulae}

In the previous chapter, we saw that for a number field $F$, the special value $\zeta_{F}^{*}(s)$ at $s=0$ contains important arithmetic information about the ring of integers $o_{F}$. A 
natural question to ask is whether the special values $\zeta_{F}^{*}(1-n)$ for integers $n \geq 2$ are also of significance.

By equation (1.4), we can see that the value $\zeta_{F}(1-n)$ for $n \geq 2$ does not vanish if and only if $F$ is a totally real number field and $n$ is even. In this case, $\zeta_{F}^{*}(1-n)=\zeta_{F}(1-n)$ is known to be a rational number ([23], [7]).

A consequence of the Main Conjecture of Iwasawa theory for all primes $p$ is the following theorem from [9]

Theorem 2.1. Let $F$ be a totally real number field and let $n \geq 2$ be an even integer. Then

$$
\zeta_{F}(1-n)= \pm \frac{h_{n}(F)}{w_{n}(F)}
$$

up to multiples of 2.

The Main Conjecture has been proven by Wiles ([31]) for odd primes $p$ and for $p=2$ if $F / \mathbb{Q}$ is abelian. For the rest of the paper we assume the Main Conjecture for $p=2$ is valid in general, in which case the 2-primary part of Theorem 2.1 is true as well.

We can now modify Corollary 1.3 to give the following bound on the discriminant.

Corollary 2.2.

$$
D^{n-\frac{1}{2}} \leq \frac{h_{n}(F)}{w_{n}(F)}\left(\frac{(2 \pi)^{n}}{2(n-1) !}\right)^{d}
$$

\subsection{Totally Positive Cohomology}

For a totally real number field $F$ and an even integer $n \geq 2$, we consider the map

$$
\phi: H_{\mathcal{M}}^{2}\left(o_{F}, \mathbb{Z}(n)\right) \longrightarrow \mathbb{D}_{\mathrm{v} \text { real }} H_{\mathcal{M}}^{2}\left(F_{v}, \mathbb{Z}(n)\right)
$$

where $v$ runs through all real infinite primes of $F$. 
Since $H_{\mathcal{M}}^{2}\left(F_{v}, \mathbb{Z}(n)\right)$ can be described by its $p$-parts, we consider the étale cohomology groups $H_{\text {ét }}^{2}\left(F_{v}, \mathbb{Z}_{p}(n)\right)$, which we have defined as projective limits of Galois cohomology groups.

For a real infinite prime $v$, we have that $\operatorname{Gal}\left(\bar{F}_{v} / F_{v}\right)=\operatorname{Gal}(\mathbb{C} / \mathbb{R})$ is a cyclic group of order 2. Hence $H^{2}\left(\operatorname{Gal}\left(\bar{F}_{v} / F_{v}\right), \mu_{p^{m}}^{\otimes n}\right)$ can be described via the equality

$$
I^{2}(G, A)=A^{G} / N_{G} A
$$

where $N_{G} A$ is the image of the norm map

$$
N_{G}: A \longrightarrow A, \quad N_{G} a=\sum_{\sigma \in G} \sigma a
$$

For even $n \geq 2, \sigma$ acts trivially on $\mu_{p^{m}}^{\otimes n}$, hence $N_{G}$ is simply multiplication by 2 . This implies that

$$
H_{\text {ét }}^{2}\left(F_{v}, \mu_{p^{m}}^{\otimes n}\right)= \begin{cases}0 & \text { for } p \text { odd } \\ \mathbb{Z} / 2 \mathbb{Z} & \text { for } p=2\end{cases}
$$

Hence,

$$
\oplus_{\mathrm{v} \text { real }} H_{\mathcal{M}}^{2}\left(F_{v}, \mathbb{Z}(n)\right) \cong \oplus_{\mathrm{v} \text { real }} \mathbb{Z} / 2 \mathbb{Z}
$$

Since $H_{\text {ét }}^{2}\left(o_{F}^{\prime}, \mathbb{Z}_{p}(n)\right) \subseteq \operatorname{ker}(\phi)$ for all odd $p$, we are thus reduced to only the 2-primary part of the map:

$$
\phi_{2}: H_{\text {ét }}^{2}\left(o_{F}^{\prime}, \mathbb{Z}_{2}(n)\right) \longrightarrow \oplus_{\mathrm{v} \text { real }} H_{\text {ét }}^{2}\left(F_{v}, \mathbb{Z}_{2}(n)\right)
$$

which will require more careful consideration.

The short exact sequence

$$
0 \longrightarrow \mathbb{Z}_{2} \longrightarrow \mathbb{Q}_{2} \longrightarrow \mathbb{Q}_{2} / \mathbb{Z}_{2} \longrightarrow 0
$$

gives rise to the long exact sequence

$$
\begin{array}{r}
\cdots \longrightarrow H_{\text {ét }}^{i-1}\left(o_{F}^{\prime}, \mathbb{Z}_{2}(n)\right) \longrightarrow H_{\text {ét }}^{i-1}\left(o_{F}^{\prime}, \mathbb{Q}_{2}(n)\right) \\
\longrightarrow H_{\text {ét }}^{i-1}\left(o_{F}^{\prime}, \mathbb{Q}_{2} / \mathbb{Z}_{2}(n)\right) \longrightarrow H_{\text {ét }}^{i}\left(o_{F}^{\prime}, \mathbb{Z}_{2}(n)\right) \longrightarrow \ldots
\end{array}
$$


For a totally real number field $F$ and an even integer $n \geq 2$, the groups $H_{\text {êt }}^{i}\left(o_{F}^{\prime}, \mathbb{Z}_{2}(n)\right)$ are finite for $i=1,2$ by the properties described in Section 2.3, so we have

$$
H_{\text {ét }}^{i}\left(o_{F}^{\prime}, \mathbb{Q}_{2}(n)\right)=H_{\text {ét }}^{i}\left(o_{F}^{\prime}, \mathbb{Z}_{2}(n)\right) \otimes \mathbb{Q}_{2}=0 .
$$

Thus the long exact sequence (2.6) gives an isomorphism

$$
H_{\text {ét }}^{1}\left(o_{F}^{\prime}, \mathbb{Q}_{2} / \mathbb{Z}_{2}(n)\right) \cong H_{\text {ét }}^{2}\left(o_{F}^{\prime}, \mathbb{Z}_{2}(n)\right)
$$

The totally positive étale cohomology groups $H_{+}^{i}\left(o_{F}^{\prime}, \bullet\right)$ defined in [2] provide a long exact sequence

$$
\begin{array}{r}
\ldots \longrightarrow H_{\text {êt }}^{1}\left(o_{F}^{\prime}, \mathbb{Q}_{2} / \mathbb{Z}_{2}(n)\right) \longrightarrow \oplus_{\mathrm{v} \text { real }} H_{\text {êt }}^{1}\left(F_{v}, \mathbb{Q}_{2} / \mathbb{Z}_{2}(n)\right) \\
\longrightarrow H_{+}^{1}\left(o_{F}^{\prime}, \mathbb{Q}_{2} / \mathbb{Z}_{2}(n)\right) \longrightarrow H_{\text {êt }}^{2}\left(o_{F}^{\prime}, \mathbb{Q}_{2} / \mathbb{Z}_{2}(n)\right) \\
\longrightarrow \oplus_{\mathrm{v} \text { real }} H_{\text {ét }}^{2}\left(F_{v}, \mathbb{Q}_{2} / \mathbb{Z}_{2}(n)\right) \longrightarrow 0 .
\end{array}
$$

In [2], it is shown that $H_{+}^{1}\left(o_{F}^{\prime}, \mathbb{Q}_{2} / \mathbb{Z}_{2}(n)\right)$ vanishes for all even $n \geq 2$. Thus (2.8) and (2.7) give the exact sequence

$$
\cdots \longrightarrow H_{\text {êt }}^{2}\left(o_{F}^{\prime}, \mathbb{Z}_{2}(n)\right) \longrightarrow \oplus_{\mathrm{v} \text { real }} H_{\text {êt }}^{2}\left(F_{v}, \mathbb{Z}_{2}(n)\right) \longrightarrow 0 .
$$

providing surjectivity of our original map

$$
\phi: H_{\mathcal{M}}^{2}\left(o_{F}, \mathbb{Z}(n)\right) \longrightarrow \oplus_{\mathrm{v} \text { real }} \mathbb{Z} / 2 \mathbb{Z} .
$$

The kernel of this map is called the positive motivic cohomology group $H_{+}^{2}\left(o_{F}, \mathbb{Z}(n)\right)$, whose order we denote by $h_{n}^{+}(F)$.

As a consequence of the surjectivity of $\phi$, we obtain the following result.

Lemma 2.3. For any totally real number field $F$ of degree $d$, and any even integer $n \geq 2$,

$$
h_{n}(F)=2^{d} \cdot h_{n}^{+}(F)
$$

This allows a restatement of the bound given in Corollary 2.2 


\section{Corollary 2.4.}

$$
D^{n-\frac{1}{2}} \leq \frac{h_{n}^{+}(F)}{w_{n}(F)}\left(\frac{(2 \pi)^{n}}{(n-1) !}\right)^{d}
$$




\section{Chapter 3}

\section{The Wild Kernel}

In this chapter we begin Section 3.1 by defining the wild kernel of a number field $F$. In the spirit of the previous chapter, we then continue by defining both the étale wild kernel and the motivic wild kernel of $F$ as well. In Section 3.2 we use the PoitouTate duality sequence to develop an equation for the order of the motivic wild kernel of $F$.

The background for the material in this chapter is taken primarily from [14] and [16].

\subsection{Definition of Wild Kernel}

For a number field $F$, the classical wild kernel $W K_{2}(F)$ fits into Moore's exact sequence

$$
0 \longrightarrow W K_{2}(F) \longrightarrow K_{2}(F) \longrightarrow \oplus_{v} \mu\left(F_{v}\right) \longrightarrow \mu(F) \longrightarrow 0
$$

where $v$ runs through all finite and real infinite primes of $F$, and $\mu(F)$ and $\mu\left(F_{v}\right)$ denote the roots of unity in $F$ and $F_{v}$ respectively.

To relate this to $K_{2}\left(o_{F}\right)$, we look at the $p$-part of $W K_{2}(F)$, denoted $W K_{2}(F)(p)$, 
which turns out to be the kernel of the map

$$
K_{2}\left(o_{F}\right)(p) \longrightarrow \oplus_{v} \mu\left(F_{v}\right)(p),
$$

where $v$ runs through all primes in $F$ above $p$ and if $p=2$, also through the real infinite primes.

The analogue of this in étale cohomology would be the higher étale wild kernel for the prime $p$, denoted by $W K_{2 n-2}^{(p)}(F)$, which is defined by

$$
W K_{2 n-2}^{(p)}(F)=\operatorname{ker}\left(H_{\text {ét }}^{2}\left(o_{F}^{\prime}, \mathbb{Z}_{p}(n)\right) \longrightarrow \oplus_{v \mid p} H_{\text {ét }}^{2}\left(F_{v}, \mathbb{Z}_{p}(n)\right),\right.
$$

where again $v$ runs through all primes in $F$ above $p$, as well as the real infinite primes if $p=2$.

In the interest of again working with a 'global' model, we define the motivic wild kernel $W K_{2 n-2}^{\mathcal{M}}(F)$ by

$$
W K_{2 n-2}^{\mathcal{M}}(F)=\prod_{p} W K_{2 n-2}^{(p)}(F)
$$

\subsection{Poitou-Tate Duality Sequence}

Let $S$ be a nonempty set of primes of a number field $F$ containing the infinite primes and let $A$ be a finite $G_{S}$ module. There exists a 9-term exact sequence called the Poitou-Tate duality sequence (cf. [16]), given by:

$$
\begin{aligned}
& 0 \longrightarrow H^{0}\left(G_{S}, A\right) \longrightarrow \oplus_{v \in S} H^{0}\left(F_{v}, A\right) \longrightarrow H^{2}\left(G_{S}, A^{\prime}\right)^{*} \longrightarrow \\
& \longrightarrow H^{1}\left(G_{s}, A\right) \longrightarrow \oplus_{v \in S} H^{1}\left(F_{v}, A\right) \longrightarrow H^{1}\left(G_{S}, A^{\prime}\right)^{*} \longrightarrow \\
& \longrightarrow H^{2}\left(G_{S}, A\right) \longrightarrow \oplus_{v \in S} H^{2}\left(F_{v}, A\right) \longrightarrow H^{0}\left(G_{S}, A^{\prime}\right)^{*} \longrightarrow 0
\end{aligned}
$$

where $A^{\prime}$ is the dualizing module of $A$, and $H^{i}\left(G_{S}, A^{\prime}\right)^{*}=H o m\left(H^{i}\left(G_{S}, A^{\prime}\right), \mathbb{Q} / \mathbb{Z}\right)$ denotes the Pontryagin dual of $H^{i}\left(G_{S}, A^{\prime}\right)$. 
For a finite prime $v \in S$, local duality yields the following isomorphism for $0 \leq i \leq 2$ :

$$
H^{i}\left(F_{v}, A\right) \cong H^{2-i}\left(F_{v}, A^{\prime}\right)^{*}
$$

We specialize now to the following situation: For a fixed prime $p$, let $S$ be the finite set of all primes above $p$ including the infinite primes if $p=2$, and let $A=\mu_{p_{m}}^{\otimes n}$. Then the dualizing $G_{S^{-}}$module of $A$ is given by $\left(\mu_{p^{m}}^{\otimes n}\right)^{\prime}=\mu_{p^{m}}^{\otimes 1-n}$ (cf. [21]), and therefore the local duality isomorphism is given by

$$
H_{\text {êt }}^{2}\left(F_{v}, \mu_{p^{m}}^{\otimes n}\right) \cong H^{0}\left(F_{v}, \mu_{p^{m}}^{\otimes 1-n}\right)^{*} .
$$

Furthermore, the last 3 terms of (3.2) now read

$$
H_{\mathrm{ett}}^{2}\left(o_{F}^{\prime}, \mu_{p^{m}}^{\otimes n}\right) \longrightarrow \oplus_{v \in S} H_{\mathrm{et}}^{2}\left(F_{v}, \mu_{p^{m}}^{\otimes n}\right) \longrightarrow H^{0}\left(F, \mu_{p^{m}}^{\otimes 1-n}\right)^{*} \longrightarrow 0
$$

Since this is an exact sequence of finite abelian groups, we can take projective limits with respect to $m$ to obtain the exact sequence

$$
H_{\text {ét }}^{2}\left(o_{F}^{\prime}, \mathbb{Z}_{p}(n)\right) \longrightarrow \oplus_{v \in S} H_{\text {ét }}^{2}\left(F_{v}, \mathbb{Z}_{p}(n)\right) \longrightarrow H^{0}\left(F, \mathbb{Q}_{p} / \mathbb{Z}_{p}(1-n)\right)^{*} \longrightarrow 0 .
$$

Here we used the fact that

$$
\begin{aligned}
\lim _{\leftarrow} H^{0}\left(F, \mu_{p^{m}}^{\otimes 1-n}\right)^{*} & \cong \lim H o m\left(H^{0}\left(F, \mu_{p^{m}}^{\otimes 1-n}\right), \mathbb{Q}_{p} / \mathbb{Z}_{p}\right) \\
& \cong H o m\left(\lim _{\longrightarrow} H^{0}\left(F, \mu_{p^{m}}^{\otimes 1-n}\right), \mathbb{Q}_{p} / \mathbb{Z}_{p}\right) \\
& \cong H o m\left(H^{0}\left(F, \mathbb{Q}_{p} / \mathbb{Z}_{p}(1-n)\right), \mathbb{Q}_{p} / \mathbb{Z}_{p}\right) \\
& \cong H^{0}\left(F, \mathbb{Q}_{p} / \mathbb{Z}_{p}(1-n)\right)^{*}
\end{aligned}
$$

By the definiton of the motivic wild kernel (3.1), the $p$-part of $W K_{2 n-2}^{\mathcal{M}}(F)$ fits 
into the exact sequence (3.4) as follows:

$$
\begin{array}{r}
0 \longrightarrow W K_{2 n-2}^{\mathcal{M}}(F)(p) \longrightarrow H_{\text {ét }}^{2}\left(o_{F}^{\prime}, \mathbb{Z}_{p}(n)\right) \\
\longrightarrow \oplus_{v \in S} H_{\text {ét }}^{2}\left(F_{v}, \mathbb{Z}_{p}(n)\right) \longrightarrow H^{0}\left(F, \mathbb{Q}_{p} / \mathbb{Z}_{p}(1-n)\right)^{*} \longrightarrow 0 .
\end{array}
$$

For a totally real number field $F$ and even integer $n \geq 2$, we can replace $H_{\text {ét }}^{2}\left(o_{F}^{\prime}, \mathbb{Z}_{p}(n)\right)$ by the $p$-part of $H_{+}^{2}\left(o_{F}, \mathbb{Z}(n)\right)$. The sequence (3.5) will remain exact if the set $S$ is reduced to include only the finite primes $v \mid p$. This yields

$$
\begin{array}{r}
0 \longrightarrow W K_{2 n-2}^{\mathcal{M}}(F)(p) \longrightarrow H_{+}^{2}\left(o_{F}, \mathbb{Z}(n)\right)(p) \longrightarrow \\
\longrightarrow \oplus_{v \mid p} H_{\text {ét }}^{2}\left(F_{v}, \mathbb{Z}_{p}(n)\right) \longrightarrow H^{0}\left(F, \mathbb{Q}_{p} / \mathbb{Z}_{p}(1-n)\right)^{*} \longrightarrow 0 .
\end{array}
$$

Since for any $t$ and any $m$ the Galois action on $\mu_{p^{m}}^{\otimes-t}$ is inverse to the action on $\mu_{p^{m}}^{\otimes t}$ we obtain

$$
\begin{aligned}
\left|H^{0}\left(F, \mathbb{Q}_{p} / \mathbb{Z}_{p}(1-n)\right)^{*}\right| & =\left|H^{0}\left(F, \mathbb{Q}_{p} / \mathbb{Z}_{p}(1-n)\right)\right| \\
& =\mid H^{0}\left(F, \mathbb{Q}_{p} / \mathbb{Z}_{p}(n-1) \mid\right. \\
& =w_{n-1}(F)(p),
\end{aligned}
$$

as well as

$$
\begin{aligned}
\left|\oplus_{v \mid p} H_{\text {ét }}^{0}\left(F_{v}, \mathbb{Q}_{p} / \mathbb{Z}_{p}(1-n)\right)^{*}\right| & =\left|\oplus_{v \mid p} H_{\text {ét }}^{0}\left(F_{v}, \mathbb{Q}_{p} / \mathbb{Z}_{p}(n-1)\right)\right| \\
& =\prod_{v \mid p} w_{n-1}\left(F_{v}\right)(p) .
\end{aligned}
$$

. Finally by (3.6), (3.7) and (3.8) we have the following formula for computing the order of the $p$-part of the motivic wild kernel:

$$
\left|W K_{2 n-2}^{M}(F)(p)\right|=w_{n-1}(F)(p) \cdot \frac{\left|H_{+}^{2}\left(o_{F}, \mathbb{Z}(n)\right)(p)\right|}{\prod_{v \mid p} w_{n-1}\left(F_{v}\right)(p)} .
$$

Let $h_{n}^{\mathcal{M}}(F)$ denote the order of the motivic wild kernel of $F$. Taking the product over all primes $p$ and using Remark 1 we obtain from (3.9): 
Theorem 3.1. For a totally real number field Fand an even integer $n \geq 2$,

$$
h_{n}^{\mathcal{M}}(F)=2 \cdot \frac{h_{n}^{+}(F)}{\prod_{p} \prod_{v \mid p} w_{n-1}\left(F_{v}\right)(p)},
$$

where $p$ runs through all prime numbers and $v$ runs through all primes in $F$ above $p$.

By Corollary 2.4, we have the following estimate of $h_{n}^{\mathcal{M}}(F)$.

\section{Corollary 3.2 .}

$$
h_{n}^{\mathcal{M}}(F) \geq D^{n-\frac{1}{2}} \cdot \frac{2 w_{n}(F)}{\prod_{p} \prod_{v \mid p} w_{n-1}\left(F_{v}\right)(p)}\left(\frac{(n-1) !}{(2 \pi)^{n}}\right)^{d} .
$$




\section{Chapter 4}

\section{Number Fields of Bounded Discriminant}

Before proceeding to the Main Theorem of the paper, we pause to explore the connection between the discriminant and degree of a number field. In section 4.1 we discuss the evolution of lower bounds for discriminants, a notion pioneered by Minkowski and advanced by Stark, Odlyzko and others. In section 4.2 we provide an application of these bounds, the process of enumerating all totally real number fields of bounded discriminant described by Voight in [27].

The material for discriminant bounds presented in this paper is taken primarily from [18] and [3].

\subsection{Odlyzko Bounds}

Let $F$ be an algebraic number field of degree $d$ over $\mathbb{Q}$ with $r_{1}$ real and $2 r_{2}$ complex conjugate fields, so that $d=r_{1}+2 r_{2}$. Let $D$ denote the discriminant of $F$.

We define the root discriminant $\delta_{F}$ of $F$ by

$$
\delta_{F}=D^{1 / d}
$$


For $F=\mathbb{Q}$, we have $D=1$, and Minkowski proved the fundamental result that $|D|>1$ for all $d>1$. Subsequently, he was able to obtain a lower bound for $|D|$ given by

$$
|D| \geq\left(\frac{\pi}{4}\right)^{2 r_{2}}\left(\frac{d^{d}}{d !}\right)^{2}
$$

This bound was improved by numerous authors, primarily through methods of geometry of numbers.

An alternative approach using analytic methods was introduced by Stark in [24], based on a relation between the discriminant of a number field and the zeros of its Dedekind zeta function. Specifically, for $s$ real, $s>1$, he gives

$$
\log |D| \geq r_{1}\left(\log \pi-\frac{\Gamma^{\prime}}{\Gamma}\left(\frac{s}{2}\right)\right)+2 r_{2}\left(\log (2 \pi)-\frac{\Gamma^{\prime}}{\Gamma}(s)\right)-\frac{2}{s}-\frac{2}{(s-1)},
$$

where $\Gamma$ denotes the Gamma function.

By taking $s=1+d^{-1 / 2}$, we obtain from (4.1) the estimate

$$
|D| \geq\left(4 \pi e^{\gamma}\right)^{r_{1}}\left(2 \pi e^{\gamma}\right)^{2 r_{2}} e^{O(d)} \quad \text { as } d \longrightarrow \infty,
$$

where $\gamma=0.5772156 \ldots$ denotes Euler's constant. This bound was then improved by Odlyzko in a series of papers, resulting in substantially improved bounds in various situations, both under the assumption of the Generalized Riemann Hypothesis and in the unconditional case.

For totally real number fields, an explicit bound is given by Poitou in [20], which reads

$$
\frac{1}{d} \log |D| \geq \gamma+\log (4 \pi)+1-8.6 d^{-2 / 3}
$$

This inequality provides a lower bound $\delta_{F} \geq B(d)$ which is increasing monotonically with $d$, i.e. $B(d+1)>B(d)$ for any degree $d \geq 2$. For small degrees, this bound has been improved upon, resulting in complete tables of bounds for degrees $d \leq 200$ which are provided in [3]. For the remainder of the paper, we define $B_{O}(d)$ to be the unconditional lower bound given in [3] for the root discriminant of a totally real number field of degree $d \leq 200$. For totally real number fields of higher degree, we define $B_{O}(d)$ to be the lower bound given by equation (4.2). By this definition, we retain the property that $B_{O}(d+1)>B_{O}(d)$ for any degree $d \geq 2$. 


\subsection{Enumeration of Totally Real Number Fields}

Our goal of characterizing the set of all totally real number fields with trivial motivic wild kernel clearly relies on the enumeration of all totally real number fields of bounded root discriminant. In fact, we must first start by assuring ourselves that this is in fact possible, i.e that there are only finitely many totally real fields of bounded root discriminant, up to isomorphism.

Given $B \in \mathbb{R}_{>0}$, let $N F(B)$ denote the set of totally real number fields $F$ with root discriminant $\delta_{F} \leq B$, up to isomorphism. For each $d \in \mathbb{Z}_{>0}$ we define a subset of $N F(B)$ given by $N F(d, B)=\{F \in N F(B) \mid[F: \mathbb{Q}]=d\}$.

Theorem 4.1. For $B<60.840, N F(B)$ is finite.

Proof. For each $d \in \mathbb{Z}_{>0}$, the set $N F(d, B)$ is finite, due to Minkowski. The Odlyzko bound (4.2) states that for $\delta<4 \pi e^{\gamma+1}$ we must have $N F(d, B)=\emptyset$ for $d$ sufficiently large. Thus the set $N F(B)$ is a finite union of finite sets for $B<4 \pi e^{\gamma+1} \approx 60.839$.

It is important to note however, that for large enough $B$, the set $N F(B)$ is in fact infinite. Infinite towers of totally real number fields with fixed root discriminant have been constructed by Martin and Martinet, with $\delta_{F} \approx 913$ and $\delta_{F} \approx 1059$ respectively.

The general method for enumerating number fields is well-known. We give an overview of the method used in [27] for enumerating totally real number fields of bounded root discriminant. We define a number field $F$ to be primitive if it contains no proper subfields $E \neq \mathbb{Q}$, and imprimitive otherwise.

\subsubsection{Enumeration Process}

Building $N S(d, B)$

Let $F=\mathbb{Q}(\alpha)$ be a field of fixed degree $d$ and fixed discriminant $D$. This fixes the degree and discriminant of the minimal polynomial of $\alpha$ as well, thus bounding 
its coefficients. Running through all discriminants $D \leq B^{d}$, the bounded coefficients yield a finite set $N S(d, B)$ of polynomials $f(x) \in \mathbb{Z}[x]$ such that every field $F \in$ $N F(d, B)$ is represented as $\mathbb{Q}[x] /(f(x))$ for some $f(x) \in N S(d, B)$. It must be noted here that this process may not capture all imprimitive fields in $N F(d, B)$, but these fields can be added later in the process.

\section{Filtering $N S(d, B)$}

The following algorithm is used to reject all polynomials in $N S(d, B)$ which do not represent totally real number fields with discriminant $D \leq B^{d}$. Fach $f \in N S(d, B)$ is tested in turn.

1. Check "easy" irreducibility; that is, discard $f$ if it is divisible by any of the following factors:

$x, x \pm 1, x \pm 2, x^{2} \pm x-1, x^{2}-2$

2. Compute the polynomial discriminant $\operatorname{disc}(f)$. If $\operatorname{disc}(f) \leq 0$, discard $f$.

3. For $f$ to represent a field $F \in N F(d, B)$, we require $B_{O}(d)^{d}<D=\operatorname{disc}(f) / a^{2} \leq$ $B^{d}$ for some $a \in \mathbb{Z}$. If no such $a^{2} \mid \operatorname{disc}(f)$ exists, discard $f$.

4. Check irreducibility. Discard $f$ if reducible.

5. Compute $o_{F}$. If $\operatorname{disc}\left(o_{F}\right)=D>B$, discard $f$.

6. Compute a small element $\alpha_{\text {red }} \in o_{F}$ such that $\mathbb{Q}\left(\alpha_{\text {red }}\right)=F$.

Add the minimal polynomial $f_{r e d}(x)$ of $\alpha_{\text {red }}$ to $N F(d, B)$, along with its discriminant $D$, if it does not already appear.

Once the list $N F(d, B)$ is complete, it is ordered by discriminant, and any isomorphic fields are discarded. Taking a union over all relevant $d$ gives a list of all primitive fields in $N F(B)$. 


\section{Adding Imprimitive fields}

Since not all imprimitive fields will be caught by the above process, they must be included separately. Any imprimitive field $F \in N F(B)$ must be an extension of a field $E \in N F(B)$, and thus can be found by the following process:

For each $E \in N F(B)$, let $f(x)$ be the minimal polynomial of an element $\alpha$ such that $F=E(\alpha)$, and the coefficients of $f(x)$ are in $o_{E}$. For a fixed relative degree $[F: E]$ and bounded discriminant, the coefficients of $f(x)$ are again bounded, and the above process applies mutatis mutandis to this relative case. Repeating this process for all necessary relative degees produces all extensions $F$ of $E$ of bounded discriminant. These fields along with their discriminants are then added to the existing list. After being resorted and divested of any isomorphic fields, the final list yields $N F(B)$.

By this process, Voight was able to enumerate all totally real number fields with root discriminant $\delta_{F} \leq 14$. He concluded that $\# N F(14)=1229$. 


\section{Chapter 5}

\section{Main Theorem}

Recall that for a totally real number field $F$ and even integer $n \geq 2$, we can determine the order of the motivic wild kernel $h_{n}^{\mathcal{M}}(F)$ by the equation given in Theorem 3.1. For each such $n$, let $\mathcal{N F}_{a}(n)$ denote the set of totally real number fields $F$ having $h_{n}^{\mathcal{M}}\left(F^{\prime}\right)=a$, where $a$ is a positive integer. Our goal is to characterize $\mathcal{N F}_{a}(n)$ for a given value of $a$. In particular, we focus on the set $\mathcal{N F}_{1}(n)$, which consists of all totally real number fields having trivial $n$-th motivic wild kernel.

Corollary 3.2 provides a bound on the order of the motivic wild kernel dependent on specific properties of the field itself. To establish a more general lower bound, we begin by forming estimations of these properties. In Section 5.1 we bound the possible values of $w_{n}(F)$, while in Section 5.2 we estimate the local conditions governing the index of $W K_{2 n-2}^{\mathcal{M}}(F)$ in $H_{+}^{2}\left(o_{F}, \mathbb{Z}(n)\right)$. Section 5.3 defines an upper bound on the root discriminant, which is then used in Section 5.4 to restrict the possible number fields having trivial motivic wild kernel. Section 5.5 provides our main theorem.

\section{$5.1 \quad$ Estimating $w_{n}(F)$}

We begin by bounding the possible values of $w_{n}(F)=\left|H^{0}(F, \mathbb{Q} / \mathbb{Z}(n))\right|$. 
Lemma 5.1. For any number field $F$ of degree $d$ and any integer $n \geq 1$ we have

$$
w_{n}(\mathbb{Q}) \leq w_{n}(F) \leq w_{d n}(\mathbb{Q}) .
$$

Proof. We have

$$
H^{0}(F, \mathbb{Q} / \mathbb{Z}(n)) \cong \prod_{p} H^{0}\left(F, \mathbb{Q}_{p} / \mathbb{Z}_{p}(n)\right)
$$

hence for the right-hand inequality it is enough to compare the orders of $H^{0}\left(F, \mathbb{Q}_{p} / \mathbb{Z}_{p}(n)\right)$ and $H^{0}\left(\mathbb{Q}, \mathbb{Q}_{p} / \mathbb{Z}_{p}(d n)\right)$ for each prime $p$.

Let $w_{n}(F)(p)=m$. Then $\operatorname{Gal}\left(F\left(\zeta_{m}\right) / F\right)=\operatorname{Gal}\left(\mathbb{Q}\left(\zeta_{m}\right) / \mathbb{Q}\left(\zeta_{m}\right) \cap F\right)$ has exponent $n$, and we have the following exact sequence of abelian groups:

$$
0 \longrightarrow \operatorname{Gal}\left(F\left(\zeta_{m}\right) / F\right) \stackrel{\alpha}{\longrightarrow} \operatorname{Gal}\left(\mathbb{Q}\left(\zeta_{m}\right) / \mathbb{Q}\right) \stackrel{\beta}{\longrightarrow} \operatorname{Gal}\left(\mathbb{Q}\left(\zeta_{m}\right) \cap F / \mathbb{Q}\right) \longrightarrow 0
$$

Consider an element $a \in G a l\left(\mathbb{Q}\left(\zeta_{m}\right) / \mathbb{Q}\right)$. We must have $d \cdot \beta(a)=0$, since $\left[\mathbb{Q}\left(\zeta_{m}\right) \cap F\right.$ : $\mathbb{Q}] \leq d$. Now, $d \cdot \beta(a)=\beta(d a)$, so $d a \in \operatorname{ker}(\beta)$. By exactness, this implies $d a=\alpha(b)$ for some $b \in \operatorname{Gal}\left(F\left(\zeta_{m}\right) / F\right)$, and hence $n \cdot d a=0$. Thus $\operatorname{Gal}\left(\mathbb{Q}\left(\zeta_{m}\right) / \mathbb{Q}\right)$ must have exponent $d n$.

For the left-hand inequality, the result follows immediately from the fact that for any field $F$ and any prime $p, \operatorname{Gal}\left(\mathbb{Q}\left(\zeta_{p^{k}}\right) / \mathbb{Q}\left(\zeta_{p^{k}}\right) \cap F\right) \subseteq \operatorname{Gal}\left(\mathbb{Q}\left(\zeta_{p^{k}}\right) / \mathbb{Q}\right)$.

\subsection{Estimating Local Factors}

Next we examine the local behaviour of our field $F$. Namely, we are concerned with the values $w_{n-1}\left(F_{v}\right)(p)$, where $v$ is a prime in $F$ above $p$. It is important to note that these values contain only the $p$-primary part of $w_{n-1}\left(F_{v}\right)$, and thus will be of the form $p^{k}$ for some integer $k \geq 0$.

For an arbitrary totally real number field $F$ of degree $d$ with discriminant $D$, we prove the following claims for an even integer $n \geq 2$ :

\section{Claim 1.}

$$
\prod_{v \mid 2} w_{n-1}\left(F_{v}\right)(2) \leq 2^{d}
$$


Proof. We have $w_{n-1}\left(F_{v}\right)(2)=w_{1}\left(F_{v}\right)(2)$, since adjoining 2-power roots of unity is a 2-power extension. Suppose there exists a totally real number field $F$ of degree $d$ such that $\prod_{v \mid 2} w_{1}\left(F_{v}\right)(2)>2^{d}$. Since there are at most $d$ primes above 2 , this implies that $w_{1}\left(F_{v}\right)(2)>2$ for some $v \mid 2$. If this is true then $\mathbb{Q}_{2}(i) \subseteq F_{v}$, and so $e(v \mid 2) \geq 2$. Thus there can be at most $d-2$ other primes above 2 , each with $w_{1}\left(F_{v}\right)(2)=2$. Thus we have $\prod_{v \mid 2} w_{n-1}\left(F_{v}\right)(2) \leq 2^{2} \cdot 2^{d-2}$, a contradiction.

Claim 2. For all odd primes $p \nmid D$,

$$
\prod_{v \mid p} w_{n-1}\left(F_{v}\right)(p)=1
$$

Proof. $n-1$ odd implies $\prod_{v \mid p} w_{n-1}\left(F_{v}\right)(p)$ can only be non-trivial if $\mathbb{Q}_{p}\left(\zeta_{p}\right) \cap F_{v}$ is non-trivial for at least one $v \mid p$. Thus $p$ must be ramified in $F$, which requires $p \mid D$.

Claim 3. For an odd prime $p$, let $n-1=p^{a} \cdot b$, where $p \nmid b$. Then for any prime $v \mid p$ in $F$,

$$
w_{n-1}\left(F_{v}\right)(p) \leq p^{a} \cdot w_{b}\left(F_{v}\right)(p) .
$$

Proof. If either $p \nmid D$ or $g c d(b, p-1)=1$, then both $w_{n-1}\left(F_{v}\right)(p)$ and $w_{b}\left(F_{v}\right)(p)$ are trivial, so the inequality holds. Suppose instead that $w_{b}\left(F_{v}\right)(p)=p^{k}$ for some $k \geq 1$. Then by definition, $\operatorname{Gal}\left(F_{v}\left(\zeta_{p^{k}}\right) / F_{v}\right)$ has exponent $b$. Now, $\operatorname{Gal}\left(F_{v}\left(\zeta_{p^{k+a}}\right) / F_{v}\left(\zeta_{p^{k}}\right)\right)$ has exponent $p^{a}$, so we can conclude that $\operatorname{Gal}\left(F_{v}\left(\zeta_{p^{k+a}}\right) / F_{v}\right)$ has exponent $p^{a} \cdot b$, as required.

Claim 4. Let $S$ be the set of all ramified primes $p \geq 3$ in $F$. Then

$$
\prod_{p \in S} \prod_{v \mid p} w_{n-1}\left(F_{v}\right)(p) \leq(n-1)^{d / 2} \cdot D
$$

Proof. Fix an odd prime $p$ and let $n-1=p^{a} \cdot b$. Suppose there are $r$ primes $v_{i}$ above $p$ in $F$ such that $w_{b}\left(F_{v_{i}}\right)(p)=p^{k_{i}}, k_{i} \geq 1$ for $i=1, \ldots, r$. Let $G=$ $\operatorname{Gal}\left(\mathbb{Q}_{p}\left(\zeta_{p^{k_{i}}}\right) / \mathbb{Q}_{p}\left(\zeta_{p^{k_{i}}}\right) \cap F_{v_{i}}\right)$, which is a subset of $\mathbb{Z} /(p-1) \mathbb{Z} \oplus \mathbb{Z} / p^{k_{i}-1} \mathbb{Z}$. If $G$ has exponent $b$, which is prime to $p$, then we must have $\left[\mathbb{Q}_{p}\left(\zeta_{p^{k_{i}}}\right) \cap F_{v_{i}}: \mathbb{Q}_{p}\right] \geq p^{k_{i}-1}$. Since $p$ is totally ramified in $\mathbb{Q}_{p}\left(\zeta_{p^{k_{i}}}\right) \cap F_{v_{i}}, e\left(v_{i} \mid p\right) \geq p^{k_{i}-1}$.

For all $k_{i}>1$, the inequality $k_{i}+1 \leq p^{k_{i}-1}$ implies that $e\left(v_{i} \mid p\right) \geq k_{i}+1$. If instead $k_{i}=1$, then $w_{b}\left(F_{v_{i}}\right)(p)=p$ implies the intersection $\mathbb{Q}_{p}\left(\zeta_{p}\right) \cap F_{v_{i}}$ is non-trivial, and 
hence $e\left(v_{i} \mid p\right) \geq 2$.

Therefore we have $e\left(v_{i} \mid p\right) \geq k_{i}+1$ for $i=1, \ldots, r$, and hence

$$
\prod_{i=1}^{r} p^{k_{i}} \mid D \text {. }
$$

For each odd prime $p$, there can be at most $r=d / 2$ ramified primes above $p$ in $F$, so we obtain by Claim 3

$$
\prod_{i=1}^{r} w_{n-1}\left(F_{v_{i}}\right)(p) \leq\left(p^{a}\right)^{d / 2} \cdot D(p) .
$$

where $D(p)$ denotes the $p$-primary part of $D$.

Taking the product over all $p \in S$ gives the desired result.

The above four claims are sufficient to bound the local factors of $F$ for any prime $p$. Taking the product over all primes gives a general bound for the local factors of $F$.

Theorem 5.2. Let $F$ be a totally real number field of degree $d$ and discriminant $D$, and let $n \geq 2$ be an even integer. Then,

$$
\prod_{p} \prod_{v \mid p} w_{n-1}(F)(p) \leq 2^{d} \cdot(n-1)^{d / 2} \cdot D .
$$

\subsection{Bounding the Root Discriminant}

For each even integer $n \geq 2$, the estimates defined in the previous section allow us to place an upper bound on the root discriminant of all totally real number fields having motivic wild kernel of a given order.

Proposition 5.3. Let $F$ be a totally real number field of degree $d$ with discriminant $D$. Let $\delta_{F}=D^{\frac{1}{d}}$, and let $n \geq 2$ be an even integer. Then a necessary condition for $h_{n}^{M}(F) \leq a$ is given by

$$
\delta_{F}<\left(\frac{2 a \cdot(2 \pi)^{n}}{(n-1)^{1 / 2} \cdot(n-2) !}\right)^{\frac{2}{2 n-3}} .
$$


Proof. Set $h_{n}^{\mathcal{M}}(F) \leq a$. By combining the bound on local factors given in Theorem 5.2 and the bound on the order of the motivic wild kernel from Corollary 3.2, we have

$$
D^{n-3 / 2} \leq \frac{a}{2 w_{n}(F)} \cdot\left(\frac{2(2 \pi)^{n}}{(n-1)^{1 / 2}(n-2) !}\right)^{d} .
$$

Taking roots gives

$$
\delta_{F} \leq\left[\left(\frac{a}{2 w_{n}(F)}\right)^{1 / d} \cdot \frac{2(2 \pi)^{n}}{(n-1)^{1 / 2}(n-2) !}\right]^{\frac{2}{2 n-3}} .
$$

For an arbitrary number field $F$, we have $2 w_{n}(F) \geq 2 w_{n}(\mathbb{Q}) \geq 48$ for all $n \geq 2$. We note that as $d \rightarrow \infty$, we have $48^{1 / d} \rightarrow 1$, so $\left(2 w_{n}(F)\right)^{1 / d}>1$. As well, $a^{1 / d} \leq a$ for any degree $d \geq 2$ so replacing $\left(\frac{a}{2 w_{n}(F)}\right)^{1 / d}$ by $a$ gives an upper bound independent of the degree.

Comparing this estimate to the Odlyzko bounds described in Section 4.1 provides our first main result.

Theorem 5.4. Let $F$ be a totally real number field such that $[F: \mathbb{Q}] \geq 2$. For any integer $a \geq 1$ there exists an even integer $N \geq 2$ such that $h_{n}^{\mathcal{M}}(F)>a$ for all even $n>N$.

Proof. We define a function on all even integers $n \geq 2$ by

$$
f_{a}(n)=\frac{2 a(2 \pi)^{n}}{(n-1)^{1 / 2}(n-2) !} .
$$

By Stirling's approximation,

$$
f_{a}(n) \leq 2 a \sqrt{2 \pi}\left(\frac{2 \pi e}{n-1}\right)^{n-\frac{1}{2}} .
$$

The function

$$
g(x)=\left(\frac{2 \pi e}{x-1}\right)^{x-\frac{1}{2}}
$$

is continuous for all $x>1$, decreasing for all $x>6.761$, and $g(x) \longrightarrow 0$ as $x \longrightarrow \infty$. Thus, for any value of $a$, there exists an even integer $N$ such that $g(N)<(2 a \sqrt{2 \pi})^{-1}$. By proposition 5.3, a necessary condition for $h_{n}^{\mathcal{M}}(F) \leq a$ is that $\delta_{F}<\left(f_{a}(n)\right)^{\frac{2}{2 n-3}}$. For all $n>N$, we have $f_{a}(n) \leq 2 a \sqrt{2 \pi} \cdot g(n)<1$, and hence $\delta_{F}<1$ as well. 
Corollary 5.5. Let $F$ be a totally real number field such that $[F: \mathbb{Q}] \geq 2$. Then $h_{n}^{\mathcal{M}}(F)>1$ for all even integers $n \geq 12$.

Proof. We have $f_{1}(n)<1$ for all even integers $n \geq 20$. The unconditional Odlyzko bounds state that for any totally real number field $F$ with $[F: \mathbb{Q}] \geq 2$, we must have $\delta_{F}>2.222$. By direct computation, we observe that $f_{1}(n)^{\frac{2}{2 n-3}}<2.222$ for even integers $12 \leq n \leq 18$ as well.

\subsection{Finding the Maximum Degree}

In the previous section, we determined that for any integer $a \geq 1$, there are finitely many even integers $n \geq 2$ such that $\mathcal{N F}_{a}(n)$ is possibly non-empty. Now we will show that for certain choices of $n$ and $a$, this set must be finite.

Proposition 5.6. For even $n \geq 4$ and $a \leq 32, \mathcal{N F}_{a}(n)$ is finite.

Proof. Let $f_{a}(n)$ be defined for all even integers $n \geq 2$ as in the proof of Theorem 5.4. Then,

$$
f_{a}(n+2)=\left(\frac{(2 \pi)^{2}}{n \sqrt{n^{2}-1}}\right) \cdot f_{a}(n)
$$

Thus, for $n \geq 8, f_{a}(n+2)<f_{a}(n)$, and hence $f_{a}(n+2)^{\frac{2}{2 n+1}}<f_{a}(n)^{\frac{2}{2 n-3}}$. By direct computation, we can see that $f_{a}(8)^{2 / 13}<f_{a}(6)^{2 / 9}<f_{a}(4)^{2 / 5}$ as well. For any value $B<60.840$, Theorem 4.1 states that the set $N F(B)$ is finite. If $a \leq 32$, $f_{a}(4)^{2 / 5}<60.785$, and hence $\mathcal{N F}_{a}(n) \subset N F(60.785)$ for all even integers $n \geq 4$.

In the case of characterizing totally real number fields with trivial motivic wild kernel, we have shown that $\mathcal{N} \mathcal{F}_{1}(n)=\emptyset$ for even integers $n \geq 12$. By Proposition 5.6, $\mathcal{N F}_{1}(n)$ is finite for $4 \leq n \leq 10$, and contains only fields of bounded degree.

For a given even integer $n \geq 2$, Proposition 5.3 states that $h_{n}^{\mathcal{M}}(F)>1$ for all totally real number fields $F$ having $\delta_{F}>B$ for some positive value $B$. If $B<60.8$, then by the unconditional Odlyzko bounds we can define a finite maximal degree 
$d_{\text {max }}(n)$ such that $B_{O}(d)>B$ for all $d>d_{\max }(n)$. Hence if $F$ is a totally real number field of degree $d>d_{\text {max }}(n)$, then $\delta_{F}>B$ and so $h_{n}^{\mathcal{M}}(F)>1$.

We begin by computing $d_{\max }(n)$ for $4 \leq n \leq 10$, which can be done almost immediately, and is termed "the easy case". Subsequently, we consider "the hard case" $n=2$, which requires more careful consideration.

\subsubsection{The Easy Case}

For $4 \leq n \leq 10, d_{\max }(n)$ can be computed by comparing the bound given in Proposition 5.3 with the Odlyzko bounds.

Table 5.1: Preliminary Degree and Root Discriminant Bounds

\begin{tabular}{|c|cccc|}
\hline $\mathrm{n}$ & 4 & 6 & 8 & 10 \\
\hline$\delta_{F}$ & $<15.194$ & $<5.582$ & $<3.343$ & $<2.380$ \\
$d_{\max }$ & 12 & 4 & 2 & 2 \\
\hline
\end{tabular}

With the maximum degree $d_{\max }(n)$ set for each value of $n$, upper bounds on the root discriminant can be calculated for each degree $d \leq d_{\max }(n)$ by reintroducing the factor of $\left(2 w_{n}(\mathbb{Q})\right)^{\frac{1}{d}}$ to the bound given in Proposition 5.3:

$$
\delta_{F} \leq\left[\left(\frac{1}{2 w_{n}(\mathbb{Q})}\right)^{1 / d} \cdot \frac{2(2 \pi)^{n}}{(n-1)^{1 / 2}(n-2) !}\right]^{\frac{2}{2 n-3}} .
$$

If we denote by $B_{d}$ the upper bound on $\delta_{F}$ for degree $d$, the set possible fields is contained in the finite set $\bigcup_{d=2}^{d_{\max }(n)} N F\left(d, B_{d}\right)$, the elements of which are enumerated by Voight (cf. Section 5.5).

For $n=8,10$, equation (5.1) states that $B_{d}<1.971$ for all $d \geq 2$, hence for any totally real number field $F$, both $h_{\mathbb{8}}^{\mathcal{M}}(F)$ and $h_{10}^{\mathcal{M}}(F)$ must be non-trivial.

The bounds $B_{d}$ for the cases $n=4$ and $n=6$ are listed in Tables 5.2 and 5.3 respectively. Table 7.2 lists the calculations performed on all possible fields, with the result being as follows: 
Table 5.2: Degree and Root Discriminant Bounds for $n=4$

\begin{tabular}{r|rr|rr}
$d$ & $\delta_{F} \leq$ & $B_{O}(d)>$ & $D \leq$ & $\# F\left(d, B_{d}\right)$ \\
\hline 2 & 4.417 & 2.223 & 19 & 5 \\
3 & 6.671 & 3.601 & 296 & 6 \\
4 & 8.195 & 5.067 & 4509 & 17 \\
5 & 9.272 & 6.523 & 68520 & 5 \\
6 & 10.068 & 7.941 & 1041084 & 13 \\
7 & 10.678 & 9.301 & 15817853 & 0 \\
8 & 11.159 & 10.596 & 240332382 & 0 \\
9 & 11.548 & 11.823 & - & 0 \\
10 & 11.869 & 12.985 & - & 0 \\
11 & 12.139 & 14.083 & - & 0 \\
12 & 12.368 & 15.121 & - & 0
\end{tabular}

Table 5.3: Degree and Root Discriminant Bounds for $n=6$

\begin{tabular}{r|rr|rr}
$d$ & $\delta_{F} \leq$ & $B_{O}(d)>$ & $D \leq$ & $\# N F\left(d, B_{d}\right)$ \\
\hline 2 & 2.589 & 2.223 & 6 & 1 \\
3 & 3.345 & 3.601 & - & 0 \\
4 & 3.802 & 5.067 & - & 0
\end{tabular}

Proposition 5.7. Let $F$ be a totally real number field of degree d. Then $h_{n}^{\mathcal{M}}(F)>1$ for even integers $n \geq 6$. There is only one totally real number field $F$ with $h_{4}^{\mathcal{M}}(F)=1$; namely, the field $F=\mathbb{Q}(\sqrt{5})$.

\subsubsection{The Hard Case}

For the case $n=2$, the original bound given by Proposition 5.3 is not able to produce a finite maximal degree $d_{\max }(2)$, and so must be refined in order to provide any information about $\mathcal{N F}_{1}(2)$. Fortunately, we can make improvements on this bound by modifying the statements of the claims given in Section 5.2.

Claim 5. For all primes $p \geq 3$,

$$
\prod_{v \mid p} w_{1}\left(F_{v}\right)(p) \leq p^{\frac{d}{p-1}} .
$$


Proof. Let $p$ be an odd prime. Suppose there are $r$ primes above $p$ in $F$ such that $w_{1}\left(F_{v_{i}}\right)(p)=p^{k_{i}}, k_{i} \geq 1$ for $i=1, \ldots, r$. Then we must have $\mathbb{Q}_{p}\left(\zeta_{p^{k_{i}}}\right) \subseteq F_{v_{i}}$ and hence $e\left(v_{i} \mid p\right) \geq p^{k_{i}-1}(p-1)$ for $i=1, \ldots, r$. We then have

$$
\begin{aligned}
d & =\sum_{i=1}^{r} e\left(v_{i} \mid p\right) f\left(v_{i} \mid p\right) \\
& \geq \sum_{i=1}^{r} p^{k_{i}-1}(p-1),
\end{aligned}
$$

which gives the result that

$$
\sum_{i=1}^{r} p^{k_{i}-1} \leq \frac{d}{p-1}
$$

The inequality $k_{i} \leq p^{k_{i}-1}$ can then be used to obtain

$$
\begin{aligned}
\prod_{i=1}^{r} p^{k_{i}} & \leq p^{\left(\sum_{i=1}^{r} p^{k_{i}-1}\right)} \\
& \leq p^{\frac{d}{p-1}}
\end{aligned}
$$

Claim 6. For all primes $p \geq 3$,

$$
\prod_{v \mid p} w_{1}\left(F_{v}\right)(p)^{p-2} \mid D(p)
$$

where $D(p)$ denotes the p-primary part of $D$.

Proof. Suppose there are $r$ primes above $p$ in $F$ such that $w_{1}\left(F_{v_{i}}\right)(p)=p^{k_{i}}, k_{i} \geq 1$ for $i=1, \ldots, r$. Then as before, $e\left(v_{i} \mid p\right) \geq p^{k_{i}-1}(p-1)$ for $i=1, \ldots, r$. Thus,

$$
\prod_{i=1}^{r} p^{p^{k_{i}-1}(p-1)-1} \mid D \text {. }
$$

The inequality $p^{k_{i}-1}(p-1)-1 \geq k_{i}(p-2)$ can then be used to obtain

$$
\prod_{i=1}^{r} p^{k_{i}(p-2)} \mid D .
$$


Through the use of these claims, we obtain a more accurate estimate of the local factors of $h_{2}^{\mathcal{M}}(F)$.

\section{Theorem 5.8.}

$$
\prod_{p} \prod_{v \mid p} w_{1}\left(F_{v}\right)(p) \leq\left(2 \cdot 3^{4 / 9} \cdot 5^{1 / 6} \cdot 7^{2 / 27}\right)^{d} \cdot D^{1 / 9}
$$

Proof. By Claim 6,

$$
\prod_{\nu \mid p} w_{1}\left(F_{v}\right)(p) \leq D(p)^{1 / 9} \quad \text { for all primes } p \geq 11,
$$

so we need only consider the primes $p=2,3,5,7$. The 2-primary part of the inequality is immediate from Claim 1, so we proceed to $p=3$.

Let $\prod_{v \mid 3} w_{1}\left(F_{v}\right)(3)=3^{k}$. By Claim $6,3^{k} \mid D(3)$, and so $3^{k / 9} \leq D(3)^{1 / 9}$. By Claim $5,3^{k} \leq 3^{d / 2}$, and so $3^{8 k / 9} \leq 3^{4 d / 9}$. Combining these two properties gives $3^{k} \leq 3^{4 d / 9}$. $D(3)^{1 / 9}$. The argument applies mutatis mutandis to the primes 5 and 7 .

With this inequality, we obtain a refinement of the bound given in Proposition 5.3 , to yield the following proposition.

Proposition 5.9. Let $F$ be a totally real number field of degree $d$ with discriminant $D$. Let $\delta_{F}=D^{\frac{1}{d}}$. Then a necessary condition for $h_{2}^{\mathcal{M}}(F)=1$ is given by

$$
\delta_{F} \leq\left(2 \cdot 3^{4 / 9} \cdot 5^{1 / 6} \cdot 7^{2 / 27} \cdot(2 \pi)^{2}\right)^{18 / 25} .
$$

This bound is now sufficient to obtain a maximum degree $d_{\max }(2)=160$ from the Odlyzko bounds. As before, this allows us to replace the factor of $\left(2 w_{2}(\mathbb{Q})\right)^{1 / d}$ in the denominator of the bound, yielding the following result.

Theorem 5.10. $h_{2}^{\mathcal{M}}(F)>1$ for all totally real number fields $F$ of degree $d \geq 118$.

Proof. An upper bound on the root discriminant of a totally real number field $F$ of degree $d$ is given by

$$
\delta_{F} \leq\left(\frac{2 \cdot 3^{4 / 9} \cdot 5^{1 / 6} \cdot 7^{2 / 27} \cdot(2 \pi)^{2}}{48^{1 / d}}\right)^{18 / 25} .
$$


For $118 \leq d \leq 160$, this yields $\delta_{F}<B_{O}(d)$.

\subsection{Enumerating the Fields}

The results given by the various bounds defined in this chapter provide a complete picture of all totally real number fields with the possibility of having trivial motivic wild kernel. Summarizing this information provides us with the main theorem of the paper.

Main Theorem. There are finitely many totally real number fields $F$ such that $h_{n}^{\mathcal{M}}(F)=1$ for some even integer $n \geq 2$.

Proof. Let $\mathcal{N F}_{1}(n)$ denote the set of totally real number fields $F$ with $h_{n}^{\mathcal{M}}(F)=1$, and let $\mathcal{N F}_{1}(d, n)$ denote the subset of $\mathcal{N F}_{1}(n)$ consisting of fields of degree $d$. Proposition 5.7 and Corollary 5.5 state that $\mathcal{N F}_{1}(n)=\emptyset$ for all $n \geq 6$, and that $\# \mathcal{N F}_{1}(4)=1$. By Theorem 5.10, $\mathcal{N F}_{1}(d, 2)=\emptyset$ for all $d \geq 118$. For each degree $d<118, \mathcal{N F}_{1}(d, n)$ is a finite set, which gives the result that

$$
\bigcup_{i=1}^{\infty} \mathcal{N F}_{1}(2 i)=\mathcal{N F}_{1}(4) \cup \bigcup_{d=2}^{117} \mathcal{N F}_{1}(d, 2)
$$

is a finite union of finite sets, hence finite itself.

Through the use of the enumeration process described in Section 5.5, we are able to compute all fields in $\mathcal{N F}_{1}(2)$ of degree $d<10$.

Corollary 5.11. There are precisely 21 fields of degree $d<10$ in $\mathcal{N F}_{1}(2)$.

Proof. For each $d<10$, we define an upper bound $B_{d}$ on the root discriminant $\delta_{F}$ by equation (5.2). $h_{2}^{\mathcal{M}}(F)$ is then computed for all $F \in N F\left(d, B_{d}\right)$. The results of these computations are shown in Table 7.1. 
In the specific case that $n=2$ and $d=2, W K_{2}^{\mathcal{M}}(F)$ is equivalent to the classical wild kernel $W K_{2}(F)$ (cf. Section 3.1). The result that there are precisely 7 real quadratic fields $F$ having $\left|W K_{2}(F)\right|=1$ was shown by Hurrelbrink in [5]. 


\section{Chapter 6}

\section{Some Special Cases}

While we have developed a clear and complete picture of $\mathcal{N F}_{1}(n)$ for even integers $n \geq 4$, the set $\mathcal{N F}_{1}(2)$ remains more ambiguous. We have established that it is a finite set consisting of number fields of degree $d \leq 117$. The results of the computations performed for $d<10$ seem to suggest that the presence of a large number of fields of higher degree is rather unlikely.

In this chapter we explore two slight variations on the original question in an attempt to better characterize this set of fields.

\subsection{Assuming GRH}

The Generalized Riemann Hypothesis (GRH) for a field $F$ is the conjecture that all zeros of the Dedekind zeta function $\zeta_{F}(s)$ found in the critical strip $0<\operatorname{Re}(s)<1$ actually lie on the critical line $\operatorname{Re}(s)=1 / 2$.

As mentioned in Section 4.1, discriminant bounds established by Odlyzko rely on a relation between the discriminant of a number field and the zeros of its Dedekind zeta function. Since the GRH restricts the possible locations of these zeros, this restriction yields improved bounds on the root discriminant, known as GRH bounds. 
In rough asymptotic form,

$$
\delta_{F} \geq(215.3325 \ldots)^{r_{1} / d}(44.7632 \ldots)^{2 r_{2} / d}-O\left((\log d)^{-2}\right) .
$$

These bounds are again increasing with the degree $d$; tables of explicit bounds for $d \leq 100$ can be found in [17].

Using these GRH bounds, we can reformulate two of our previous results.

Proposition 6.1. Under the assumption of the Generalized Riemann Hypothesis, $\mathcal{N F}_{a}(n)$ is finite for even $n \geq 4$ and $a \leq 756$.

Proof. By equation (6.1), the set $N F(B)$ is finite for any value $B<215.333$. Let $f_{a}(n)$ be defined for all even integers $n \geq 2$ as in the proof of Theorem 5.4. Then, for $a \leq 756$, we have $f_{a}(4)^{2 / 5}<215.314$, and hence $\mathcal{N F}_{a}(n) \subset N F(215.314)$ for all even integers $n \geq 4$.

Proposition 6.2. If the Generalized Riemann Hypothesis is assumed to be true, then $h_{2}^{\mathcal{M}}(F)>1$ for all totally real number fields $F$ of degree $d \geq 47$.

Proof. Comparing the bound given in Proposition 5.9 to the GRH bounds gives the result that $h_{2}^{\mathcal{M}}(F)>1$ for all $d>50$. By replacing the factor of $48^{1 / d}$ in the denominator of the bound, we can reduce the maximal degree to $d=46$.

\subsection{Restricting Local Factors}

All of our estimates so far have taken into account the possibility that $F$ contains ramified local cyclotomic extensions, i.e. for some odd prime $p$, there exists some $v \mid p$ such that $\mathbb{Q}_{p}\left(\zeta_{p}\right) \subseteq F_{v}$, or there exists some $v \mid 2$ such that $\mathbb{Q}(i) \subseteq F_{v}$. This is equivalent to the condition that for some odd prime $p$,

$$
\prod_{v \mid p} w_{1}\left(F_{v}\right)(p)>1
$$

or that for $p=2$,

$$
\prod_{v \mid 2} w_{1}\left(F_{v}\right)(p)>2^{\#(v \mid 2)}
$$


where \#(v|2) denotes the number of primes above 2 in $F$.

While this would not appear to be a rare occurrence, we observe from the claims made in Section 5.4 that for an odd prime $p$ :

$$
\prod_{v \mid p} w_{1}\left(F_{v}\right)(p)>1 \quad \Longleftrightarrow \quad p \leq d+1, p^{p-2} \mid D, \text { and } \mathbb{Q}_{p}\left(\zeta_{p}\right) \subseteq F_{v} \text { for some } v \mid p,
$$

and for the prime $p=2$,

$$
\prod_{v \mid 2} w_{1}\left(F_{v}\right)(2)>2^{\#(v \mid 2)} \Longleftrightarrow 2 \mid D \text { and } \mathbb{Q}_{2}(i) \subseteq F_{v} \text { for some } v \mid 2 \text {. }
$$

Thus $F$ may contain ramified local cyclotomic extensions for only finitely many primes $p$, all of which must be contained in the interval $[2, d+1]$. It is therefore not surprising that of the 21 number fields listed in Table 7.1, only 7 contain a ramified local cyclotomic extension, and we find no fields containing such extensions for multiple primes.

An interesting case to consider arises if we assume $F$ contains no ramified local cyclotomic extensions. Specifically, let $F$ be totally real number field having the properties that for all odd primes $p, \prod_{v \mid p} w_{1}\left(F_{v}\right)(p)=1$, and $\prod_{v \mid 2} w_{1}\left(F_{v}\right)(2)=2^{\#(v \mid 2)}$.

Under this assumption, the index of $W K_{2}^{\mathcal{M}}(F)$ in $H_{+}^{2}\left(o_{F}, \mathbb{Z}(2)\right)$ is given by

$$
\frac{h_{2}^{+}(F)}{h_{2}^{\mathcal{M}}(F)}=2^{\#(v \mid 2)-1} \text {. }
$$

As before, we wish to bound the root discriminant of any such fields having trivial motivic wild kernel. The following bound is obtained via the functional equation of $\zeta_{F}(s)$.

Proposition 6.3. Let $F$ be a totally real number field of degree d with root discriminant $\delta_{F}=D^{\frac{1}{d}}$. If it is assumed that for all odd primes $p$ and all $v \mid p, \mathbb{Q}_{p}\left(\zeta_{p}\right) \nsubseteq F_{v}$ and that $\mathbb{Q}_{2}(i) \nsubseteq F_{v}$ for all $v \mid 2$, then a necessary condition for $h_{2}^{\mathcal{M}}(F)=1$ is given by

$$
\delta_{F} \leq\left(2(2 \pi)^{2}\right)^{2 / 3}
$$


Proof. Combining Lemma 1.2 and Theorem 3.1, we obtain the following equality for a totally real number field $F$ having $h_{2}^{\mathcal{M}}(F)=1$.

$$
D^{\frac{3}{2}}=\left(\prod_{p \mid D} \prod_{v \mid p} w_{1}\left(F_{v}\right)(p)\right) \frac{(2 \pi)^{2 d}}{2 w_{2}(F)} \cdot \frac{1}{\zeta_{F}(2)}
$$

Under the necessary assumptions on $F$,

$$
\prod_{v \mid p} w_{1}\left(F_{v}\right)(p)=1
$$

for all odd primes $p$. However, for $p=2, w_{1}\left(F_{v}\right)(2)=2$ for each $v \mid 2$. Since there are at most $d$ primes above 2 , we have

$$
\prod_{v \mid 2} w_{1}\left(F_{v}\right)(2) \leq 2^{d}
$$

Substituting this estimate for the local factors in equation (6.2) yields,

$$
\delta_{F}=\left(\frac{2(2 \pi)^{2}}{\left(2 w_{2}(F) \cdot \zeta_{F}(2)\right)^{1 / d}}\right)^{2 / 3}
$$

The result follows immediately from the inequality $2 w_{2}(F) \cdot \zeta_{F}(2)>1$.

This bound is sufficient to yield a maximal degree $d_{\max }(2)$, which can then be improved using equation (6.3), resulting in $d_{\max }(2)=11$.

We make the following claim concerning the value of $\zeta_{F}(2)$.

Claim 7. If $F$ contains no ramified local cyclotomic extensions and $\prod_{v \mid 2} w_{1}\left(F_{v}\right)(2)=$ $2^{d}$, then $\zeta_{F}(2) \geq(4 / 3)^{d}$.

Proof. Suppose $\prod_{v \mid 2} w_{1}\left(F_{v}\right)(2)=2^{d}$. Under the assumptions on $F$, this implies that 2 splits completely in $F$. Therefore the Euler product expansion of $\zeta_{F}(2)$ contains the factor $\left(1-\frac{1}{2^{2}}\right)^{-d}=\left(\frac{4}{3}\right)^{d}$. Since all Fuler factors of $\zeta_{F}(2)$ are $>1$, the result follows. 
Using this claim, we can make a slight improvement on the previous bound to yicld our final result.

Theorem 6.4. There are precisely 14 fields in $\mathcal{N F}_{1}(2)$ containing no ramified local cyclotomic extensions.

Proof. If 2 splits completely in $F$, we obtain by Claim 7 and equation (6.3)

$$
\delta_{F} \leq\left(\frac{6 \pi^{2}}{48^{1 / d}}\right)^{2 / 3}
$$

which yields $d_{\max }(2)=8$ in this case. On the other hand, if 2 does not split completely in $F$, then

$$
\prod_{v \mid 2} w_{1}\left(F_{v}\right)(2) \leq 2^{d-1},
$$

and $\zeta_{F}(2) \geq 1$, so the inequality in (6.3) can be rewritten as

$$
\delta_{F} \leq\left(\frac{2(2 \pi)^{2}}{96^{1 / d}}\right)^{2 / 3},
$$

which yields $d_{\max }(2)=9$. All possible fields are therefore contained in the list enumerated in Corollary 5.11 and displayed in Table 7.1. 


\section{Chapter 7}

\section{Computations}

We have seen in the previous chapter that for any positive integer $a \leq 32$ and even integer $n \geq 4, \mathcal{N F}_{a}(n)$ is a finite set, and hence can be determined by computational means. In Section 7.1 we provide an algorithm which can be used for computing such lists. Section 7.2 displays results obtained through the use of this algorithm.

All computations shown here were performed in Sage [25] and PARI/GP [19]. The enumeration of totally real fields of bounded discriminant was performed using the Sage function enumerate_totallyreal_fields_all() developed by Voight [27]. The identification of local fields was accomplished using the interactive PARI/GP database developed by Jones and Roberts described in [6].

\subsection{Enumerating $\mathcal{N F}_{a}(n)$}

For a positive integer $a \leq 32$ and an even integer $n \geq 4$, the set $\mathcal{N F}_{a}(n)$ can be computed using the following process. 


\section{Building the List}

The following algorithm will produce a set $S$ of totally real number fields which contains all possible fields in $\mathcal{N F}_{a}(n)$. We initialize $S$ to be the empty set.

1. Compute $d_{\text {max }}(n)$.

2. For each $2 \leq d \leq d_{\max }(n)$,

- Compute an upper bound on the root discriminant $\delta_{F}(n)<B_{d}$.

- Add $N F\left(d, B_{d}\right)$ to the set $S$.

\section{Filtering the List}

The following algorithm is used to reject all number fields $F \in S$ such that $h_{n}^{\mathcal{M}}(F) \neq a$. Each field is tested in turn.

1. Evaluate $\zeta_{F}(1-n)$.

2. Compute $w_{n}(F)$.

3. Let $x=\frac{w_{n}(F) \cdot \zeta_{F}(1-n)}{2^{d-1}}$.

4. If $a \nmid x$, discard $F$.

5. If there exists an odd prime $p \mid \frac{x}{a}$ such that $p \nmid D$, discard $F$.

6. If there exists an odd prime $p \mid \frac{x}{a}$ such that $(p-1)>d(n-1)$, discard $F$.

7. Let $2^{k}$ be the highest power of 2 dividing $\frac{x}{a}$. If $k>d$, discard $F$.

8. For each prime $p \mid \frac{x}{a}$ :

- Compute $\prod_{v \mid p} w_{n-1}\left(F_{v}\right)(p)=p^{k}$

- Let $x:=\frac{x}{p^{k}}$

If $x \neq a$, discard $F$. 
The above algorithm can be used to compute the elements of $\mathcal{N} \mathcal{F}_{1}(2)$ of small degree as well. Table 7.1 lists the results obtained via this algorithm for degree $d<10$. Table 7.2 lists the results for the case $n=4$, including the fields rejected by the filtering process.

\subsection{Results}

Table 7.1: Totally Real Number Fields of degree $d<10$ with $h_{2}^{\mathcal{M}}(F)=1$

\begin{tabular}{|c|c|c|c|c|}
\hline $\mathrm{D}$ & $f(x)$ & $w_{2}(F)$ & $\zeta_{F}(-1)$ & local factors \\
\hline 5 & $x^{2}-x-1$ & 120 & $\frac{1}{30}$ & - \\
\hline 8 & $x^{2}-2$ & 48 & $\frac{1}{12}$ & - \\
\hline 12 & $x^{2}-3$ & 24 & $\frac{1}{6}$ & - \\
\hline 13 & $x^{2}-x-3$ & 24 & $\frac{1}{6}$ & - \\
\hline 17 & $x^{2}-x-4$ & 24 & $\frac{1}{3}$ & - \\
\hline 24 & $x^{2}-6$ & 24 & $\frac{1}{2}$ & $F_{v} \cong \mathbb{Q}_{3}\left(\zeta_{3}\right)$ \\
\hline 33 & $x^{2}-x-8$ & 24 & 1 & $F_{v} \cong \mathbb{Q}_{3}\left(\zeta_{3}\right)$ \\
\hline 49 & $x^{3}-x^{2}-2 x+1$ & 168 & $\frac{1}{21}$ & - \\
\hline 81 & $x^{3}-3 x-1$ & 72 & $\frac{1}{9}$ & - \\
\hline 148 & $x^{3}-x^{2}-3 x+1$ & 24 & $\frac{1}{3}$ & - \\
\hline 169 & $x^{3}-x^{2}-4 x-1$ & 24 & $\frac{1}{3}$ & - \\
\hline 229 & $x^{3}-4 x-1$ & 24 & $\frac{2}{3}$ & - \\
\hline 316 & $x^{3}-x^{2}-4 x+2$ & 24 & $\frac{4}{3}$ & $F_{v} \cong \mathbb{Q}_{2}(i)$ \\
\hline 321 & $x^{3}-x^{2}-4 x+1$ & 24 & 1 & $F_{v} \cong \mathbb{Q}_{3}\left(\zeta_{3}\right)$ \\
\hline 725 & $x^{4}-x^{3}-3 x^{2}+x+1$ & 120 & $\frac{2}{15}$ & - \\
\hline 1957 & $x^{4}-4 x^{2}-x+1$ & 24 & $\frac{2}{3}$ & - \\
\hline 2000 & $x^{4}-5 x^{2}+5$ & 120 & $\frac{2}{3}$ & $F_{v} \cong \mathbb{Q}_{5}\left(\zeta_{5}\right)$ \\
\hline 2304 & $x^{4}-4 x^{2}+1$ & 48 & 1 & $F_{v} \supset \mathbb{Q}_{3}\left(\zeta_{3}\right)$ \\
\hline 2777 & $x^{4}-x^{3}-4 x^{2}+x+2$ & 24 & $\frac{4}{3}$ & - \\
\hline 3981 & $x^{4}-x^{3}-4 x^{2}+2 x+1$ & 24 & 2 & $F_{v} \cong \mathbb{Q}_{3}\left(\zeta_{3}\right)$ \\
\hline 24217 & $x^{5}-5 x^{3}-x^{2}+3 x+1$ & 24 & $\frac{4}{3}$ & - \\
\hline
\end{tabular}


Table 7.2: Computations for the case $n=4$

\begin{tabular}{|c|c|c|c|c|c|}
\hline $\mathrm{D}$ & $f(x)$ & $w_{4}(F)$ & $\zeta_{F}(-3)$ & local factors & $h_{4}^{\mathcal{M}}(F)$ \\
\hline 5 & $x^{2}-x-1$ & 240 & $\frac{1}{60}$ & - & 1 \\
\hline 8 & $x^{2}-2$ & 480 & $\frac{11}{120}$ & - & 11 \\
\hline 12 & $x^{2}-3$ & 240 & $\frac{23}{60}$ & - & 23 \\
\hline 13 & $x^{2}-x-3$ & 240 & $\frac{29}{60}$ & - & 29 \\
\hline 17 & $x^{2}-x-4$ & 240 & $\frac{41}{30}$ & - & 41 \\
\hline 49 & $x^{3}-x^{2}-2 x+1$ & 1680 & $\frac{79}{210}$ & - & 79 \\
\hline 81 & $x^{3}-3 x-1$ & 720 & $\frac{199}{90}$ & - & 199 \\
\hline 148 & $x^{3}-x^{2}-3 x+1$ & 240 & $\frac{577}{30}$ & - & 577 \\
\hline 169 & $x^{3}-x^{2}-4 x-1$ & 3120 & $\frac{11227}{390}$ & - & 11227 \\
\hline 229 & $x^{3}-4 x-1$ & 240 & $\frac{1333}{15}$ & - & 1333 \\
\hline 257 & $x^{3}-x^{2}-4 x+3$ & 240 & $\frac{1891}{15}$ & - & 3782 \\
\hline 725 & $x^{4}-x^{3}-3 x^{2}+x+1$ & 240 & $\frac{541}{15}$ & - & 541 \\
\hline 1125 & $x^{4}-x^{3}-4 x^{2}+4 x+1$ & 240 & $\frac{2522}{15}$ & - & 2522 \\
\hline 1600 & $x^{4}-6 x^{2}+4$ & 480 & $\frac{17347}{30}$ & - & 17347 \\
\hline 1957 & $x^{4}-4 x^{2}-x+1$ & 240 & $\frac{3541}{3}$ & - & 17705 \\
\hline 2000 & $x^{4}-5 x^{2}+5$ & 240 & $\frac{3793}{3}$ & $F_{v} \cong \mathbb{Q}_{5}\left(\zeta_{5}\right)$ & 3793 \\
\hline 2048 & $x^{4}-4 x^{2}+2$ & 960 & $\frac{87439}{60}$ & - & 87439 \\
\hline 2225 & $x^{4}-x^{3}-5 x^{2}+2 x+4$ & 240 & $\frac{9202}{5}$ & - & 13803 \\
\hline 2304 & $x^{4}-4 x^{2}+1$ & 480 & $\frac{22011}{10}$ & $F_{v} \supset \mathbb{Q}_{3}\left(\zeta_{3}\right)$ & 22011 \\
\hline 2525 & $x^{4}-2 x^{3}-4 x^{2}+5 x+5$ & 240 & $\frac{42787}{15}$ & - & 42787 \\
\hline 2624 & $x^{4}-2 x^{3}-3 x^{2}+2 x+1$ & 480 & $\frac{32681}{10}$ & - & 98043 \\
\hline
\end{tabular}


Table 7.2: Computations for the case $n=4$, continued

\begin{tabular}{|c|c|c|c|c|c|}
\hline $\mathrm{D}$ & $f(x)$ & $w_{4}(F)$ & $\zeta_{F}(-3)$ & local factors & $h_{4}^{\mathcal{M}}(F)$ \\
\hline 2777 & $x^{4}-x^{3}-4 x^{2}+x+2$ & 240 & $\frac{63478}{15}$ & - & 31739 \\
\hline 3600 & $x^{4}+2 x^{3}-7 x^{2}-8 x+1$ & 240 & $\frac{49404}{5}$ & $F_{v} \supset \mathbb{Q}_{2}(i), F_{v} \supset \mathbb{Q}_{3}\left(\zeta_{3}\right)$ & 24702 \\
\hline 3981 & $x^{4}-x^{3}-4 x^{2}+2 x+1$ & 240 & $\frac{70953}{5}$ & $F_{v} \cong \mathbb{Q}_{3}\left(\zeta_{3}\right)$ & 70953 \\
\hline 4205 & $x^{4}-x^{3}-5 x^{2}-x+1$ & 240 & $\frac{84937}{5}$ & - & 254811 \\
\hline 4225 & $x^{4}-9 x^{2}+4$ & 240 & $\frac{260536}{15}$ & - & 130268 \\
\hline 4352 & $x^{4}-6 x^{2}-4 x+2$ & 480 & $\frac{61204}{3}$ & - & 612040 \\
\hline 4400 & $x^{4}-7 x^{2}+11$ & 240 & $\frac{300017}{15}$ & - & 300017 \\
\hline 14641 & $x^{5}-x^{4}-4 x^{3}+3 x^{2}+3 x-1$ & 2640 & $\frac{1695622}{165}$ & - & 847811 \\
\hline 24217 & $x^{5}-5 x^{3}-x^{2}+3 x+1$ & 240 & $\frac{898526}{15}$ & - & 449263 \\
\hline 36497 & $x^{5}-2 x^{4}-3 x^{3}+5 x^{2}+x-1$ & 240 & $\frac{763388}{3}$ & - & 1908470 \\
\hline 38569 & $x^{5}-5 x^{3}+4 x-1$ & 240 & $\frac{4576108}{15}$ & - & 2288054 \\
\hline 65657 & $x^{5}-x^{4}-5 x^{3}+2 x^{2}+5 x+1$ & 240 & $\frac{29852518}{15}$ & - & 14926259 \\
\hline 300125 & $x^{6}-x^{5}-7 x^{4}+2 x^{3}+7 x^{2}-2 x-1$ & 1680 & $\frac{323983108}{105}$ & - & 80995777 \\
\hline 371293 & $x^{6}-x^{5}-5 x^{4}+4 x^{3}+6 x^{2}-3 x-1$ & 3120 & $\frac{1267169036}{195}$ & - & 316792259 \\
\hline 434581 & $x^{6}-2 x^{5}-4 x^{4}+5 x^{3}+4 x^{2}-2 x-1$ & 1680 & $\frac{1183699028}{105}$ & - & 295924757 \\
\hline 453789 & $x^{6}-x^{5}-6 x^{4}+6 x^{3}+8 x^{2}-8 x+1$ & 1680 & $\frac{196804168}{15}$ & $F_{v} \cong \mathbb{Q}_{7}\left(\zeta_{7}\right)$ & 49201042 \\
\hline 485125 & $x^{6}-2 x^{5}-4 x^{4}+8 x^{3}+2 x^{2}-5 x+1$ & 240 & $\frac{248556284}{15}$ & - & 62139071 \\
\hline 592661 & $x^{6}-x^{5}-5 x^{4}+4 x^{3}+5 x^{2}-2 x-1$ & 240 & $\frac{167017444}{5}$ & - & 125263083 \\
\hline 703493 & $x^{6}-2 x^{5}-5 x^{4}+11 x^{3}+2 x^{2}-9 x+1$ & 1680 & $\frac{2129657668}{35}$ & - & 1597243251 \\
\hline 722000 & $x^{6}-x^{5}-6 x^{4}+7 x^{3}+4 x^{2}-5 x+1$ & 240 & $\frac{334446268}{5}$ & - & 250834701 \\
\hline 810448 & $x^{6}-3 x^{5}-2 x^{4}+9 x^{3}-5 x+1$ & 240 & $\frac{1503507364}{15}$ & - & 375876841 \\
\hline 820125 & $x^{6}-9 x^{4}-4 x^{3}+9 x^{2}+3 x-1$ & 720 & $\frac{4684354132}{45}$ & - & 1171088533 \\
\hline 905177 & $x^{6}-x^{5}-7 x^{4}+9 x^{3}+7 x^{2}-9 x-1$ & 1680 & $\frac{15443870528}{105}$ & - & 1930483816 \\
\hline 966125 & $x^{6}-x^{5}-6 x^{4}+4 x^{3}+8 x^{2}-1$ & 240 & $\frac{2774660768}{15}$ & - & 693665192 \\
\hline 980125 & $x^{6}-x^{5}-6 x^{4}+6 x^{3}+7 x^{2}-5 x-1$ & 240 & $\frac{2.913920288}{15}$ & - & 728480072 \\
\hline
\end{tabular}




\section{Bibliography}

[1] J. W. S. Cassels and A. Fröhlich, editors. Algebraic number theory. Academic Press, London, 1967.

[2] T. Chinburg, M. Kolster, G. Pappas, and V. Snaith. Galois structure of $K$-groups of rings of integers. K-Theory, 14(4):319-369, 1998.

[3] F. Diaz y Diaz. Tables minorant la racine $n$-ième du discriminant d'un corps de degré $n$, volume 6 of Publications Mathématiques d'Orsay 80. Université de Paris-Sud Département de Mathématique, Orsay, 1980.

[4] D. S. Dummit and R. M. Foote. Abstract Algebra. John Wiley \& Sons Inc., Hoboken, NJ, third edition, 2004.

[5] J. Hurrelbrink. On the wild kernel. Arch. Math., 40(4):316-318, 1983.

[6] J. W. Jones and D. P. Roberts. A database of local fields. Journal of Symbolic Computation, 41(1):80 - 97, 2006.

[7] H. Klingen. Über die Werte der Dedekindschen Zetafunktion. Math. Ann., 145:265-272, 1961/1962.

[8] M. Kolster. Higher relative class number formulae. Math. Ann., 323(4):667-692, 2002.

[9] M. Kolster. $K$-theory and arithmetic. In Contemporary developments in algebraic K-theory, ICTP Lect. Notes, XV, pages 191-258 (electronic). Trieste, 2004.

[10] M. Kolster and A. Movahhedi. Galois co-descent for étale wild kernels and capitulation. Ann. Inst. Fourier, 50(1):35-65, 2000. 
[11] S. Lichtenbaum. Values of zeta-functions, étale cohomology, and algebraic $K$ theory. In Algebraic K-theory, II: "Classical" algebraic K-theory and connections with arithmetic (Proc. Conf., Battelle Memorial Inst., Seattle, Wash., 1972), pages 489-501. Lecture Notes in Math., Vol. 342. Springer, Berlin, 1973.

[12] J. Martinet. Petits discriminants des corps de nombres. In Number theory days, 1980 (Exeter, 1980), volume 56 of London Math. Soc. Lecture Note Ser., pages 151-193. Cambridge Univ. Press, Cambridge, 1982.

[13] J. S. Milne. Étale cohomology, volume 33 of Princeton Mathematical Series. Princeton University Press, Princeton, N.J., 1980.

[14] J. Milnor. Introduction to algebraic K-theory. Princeton University Press, Princeton, N.J., 1971. Annals of Mathematics Studies, No. 72.

[15] J. Neukirch. Algebraic number theory, volume 322 of Grundlehren der Mathematischen Wissenschaften. Springer-Verlag, Berlin, 1999.

[16] J. Neukirch, A. Schmidt, and K. Wingberg. Cohomology of number fields, volume 323 of Grundlehren der Mathematischen Wissenschaften. Springer-Verlag, Berlin, 2000.

[17] A. M. Odlyzko. Discriminant bounds. unpublished, available from http://www.dtc.umn.edu/ odlyzko/unpublished/index.html,.

[18] A. M. Odlyzko. Bounds for discriminants and related estimates for class numbers, regulators and zeros of zeta functions: a survey of recent results. Sém. Théor. Nombres Bordeaux (2), 2(1):119-141, 1990.

[19] The PARI Group, Bordeaux. PARI/GP, version 2.3.5, 2008. available from http://pari.math.u-bordeaux.fr/.

[20] G. Poitou. Sur les petits discriminants. In Séminaire Delange-Pisot-Poitou, 18e année: (1976/77), Théorie des nombres, Fasc. 1 (French), pages Exp. No. 6, 18. Secrétariat Math., Paris, 1977.

[21] J. Rognes and C. Weibel. Two-primary algebraic $K$-theory of rings of integers in number fields. J. Amer. Math. Soc., 13(1):1-54, 2000. Appendix A by Manfred Kolster.

[22] J.-P. Serre. Local fields, volume 67 of Graduate Texts in Mathematics. SpringerVerlag, New York, 1979. Translated from the French by Marvin Jay Greenberg. 
[23] C. L. Siegel. Berechnung von Zetafunktionen an ganzzahligen Stellen. Nachr. Akad. Wiss. Göttingen Math.-Phys. Kl. II, 1969:87-102, 1969.

[24] H. M. Stark. A complete determination of the complex quadratic fields of classnumber one. Michigan Math. J., 14:1-27, 1967.

[25] W. Stein et al. Sage Mathematics Software (Version 4.4.1). The Sage Development Team, 2009. http://www. sagemath. org.

[26] J. Tate. Symbols in arithmetic, 1970. Lecture at the International Congress in Nice.

[27] J. Voight. Enumeration of totally real number fields of bounded root discriminant. In Algorithmic number theory, volume 5011 of Lecture Notes in Comput. Sci., pages 268-281. Springer, Berlin, 2008.

[28] J. Voight. The Gauss higher relative class number problem. Ann. Sci. Math. Québec, 32(2):221-232, 2008.

[29] L. C. Washington. Introduction to cyclotomic fields, volume 83 of Graduate Texts in Mathematics. Springer-Verlag, New York, second edition, 1997.

[30] M. Watkins. Class numbers of imaginary quadratic fields. Math. Comp., 73(246):907-938 (electronic), 2004.

[31] A. Wiles. The Iwasawa conjecture for totally real fields. Ann. of Math. (2), 131(3):493-540, 1990. 
